\title{
Existence of extremal solutions for discontinuous Stieltjes differential equations
}

Rodrigo López Pouso ${ }^{1}$ and Ignacio Márquez Albés ${ }^{1 *}$ (D)

"Correspondence:

ignacio.marquez@usc.es

'Departamento de Estatística, Análise Matemática e Optimización, Faculty of Mathematics, Universidade de Santiago de Compostela, Santiago de Compostela, Spain

\section{Springer}

\begin{abstract}
Stieltjes differential equations, which contain equations with impulses and equations on time scales as particular cases, simply consist in replacing usual derivatives by derivatives with respect to a nondecreasing function. In this paper we prove new results for the existence of extremal solutions for discontinuous Stieltjes differential equations. In particular, we prove that the pointwise infimum of upper solutions of a Stieltjes differential equation is the minimal solution under certain hypotheses. These results can be adapted to the context of both difference equations and impulsive differential equations.
\end{abstract}

MSC: 34A36; 34K05

Keywords: Upper solution; Ordinary differential equations; Impulsive differential equations; Dynamic equations; Time scales

\section{Introduction}

Let us consider the initial value problem

$$
x_{g}^{\prime}(t)=f(t, x(t)), \quad t \in I=[0,1], x(0)=0,
$$

where $x_{g}^{\prime}(t)$ denotes the Stieltjes derivative of the unknown with respect to a nondecreasing and left-continuous function $g: \mathbb{R} \longrightarrow \mathbb{R}$ as introduced in [7].

The aim of this paper is to replicate the results obtained in [4] for ODEs in the more general context of Stieltjes differential equations. That is, to solve as satisfactorily as possible the following problem: to find the weakest sufficient conditions over the right-hand side $f \in \mathcal{L}_{g}^{1}(I)$ so that the minimal solution solution is the least upper solution and the maximal one is the greatest lower solution. In [5] we can find some results regarding the existence of extremal solutions of this type of equation in the presence of a pair of well-ordered lower and upper solutions. In [6] the authors followed this line of research working in the context of measure differential equations, and then adapted the results obtained to the framework of Stieltjes differential equations. Therefore, this paper complements, in a sense, the study initiated in these papers.

We have organised the paper as follows. In Sect. 2, we present the basic definitions and results required for this paper. In Sect. 3, we are looking for some necessary conditions

(c) The Author(s) 2020. This article is licensed under a Creative Commons Attribution 4.0 International License, which permits use, sharing, adaptation, distribution and reproduction in any medium or format, as long as you give appropriate credit to the original author(s) and the source, provide a link to the Creative Commons licence, and indicate if changes were made. The images or other third party material in this article are included in the article's Creative Commons licence, unless indicated otherwise in a credit line to the material. If material is not included in the article's Creative Commons licence and your intended use is not permitted by statutory regulation or exceeds the permitted use, you will need to obtain permission directly from the copyright holder. To view a copy of this licence, visit http://creativecommons.org/licenses/by/4.0/. 
for $f$ that assure that the infimum of all upper solutions of (1.1) is a solution. Then, in Sect. 4 we obtain a new existence result from those proved in the previous section. Finally, in Sect. 5, we present a result that guarantees the existence of the extremal solutions for Stieltjes differential equations. We then adapt the results obtained to difference equations and impulsive differential equations.

As a final comment, note that in this paper we work on the interval $I=[0,1]$ and the initial condition $x(0)=0$ for simplicity, but the results are true for any other interval $\tilde{I}=$ $[a, b]$ and any other initial condition $x(a)=x_{a}, x_{a} \in \mathbb{R}$, by doing the obvious changes.

\section{Preliminaries}

Let $g: \mathbb{R} \rightarrow \mathbb{R}$ be a nondecreasing and left-continuous function. In order to recall the definition of the Stieltjes derivative of a function with respect to $g$ (or simply the $g$-derivative of a function) as presented in [7], we need to define the sets

$$
C_{g}=\{s \in \mathbb{R}: g \text { is constant on }(s-\varepsilon, s+\varepsilon) \text { for some } \varepsilon>0\},
$$

and $D_{g}=\{t \in \mathbb{R}: \Delta g(t)>0\}$, where $\Delta g(t)=g\left(t^{+}\right)-g(t)$ and $g\left(t^{+}\right)$denotes the limit of $g$ at $t$ from the right. Now the $g$-derivative of a function $x: I \longrightarrow \mathbb{R}$ at a point $t \in I \backslash C_{g}$ is

$$
x_{g}^{\prime}(t)= \begin{cases}\lim _{s \rightarrow t} \frac{x(s)-x(t)}{g(s)-g(t)} & \text { if } t \notin D_{g}, \\ \lim _{s \rightarrow t^{+}} \frac{x(t)-x(t)}{g\left(s^{+}\right)-g(t)} & \text { if } t \in D_{g} \text { and } t<1,\end{cases}
$$

provided that the corresponding limit exists. Note that, for a point $t \in D_{g}, x_{g}^{\prime}(t)$ exists if and only if $x\left(t^{+}\right)$exists.

Notice that we do not define $g$-derivatives at points $t \in C_{g}$, nor it is necessary because $C_{g}$ is a null-measure set for $\mu_{g}$ (the Lebesgue-Stieltjes measure induced by $g$ ), see [7, Proposition 2.5]. Therefore, the differential equation in (1.1) is not really defined for $t \in I \cap C_{g}$.

The following result, the fundamental theorem of calculus for the Lebesgue-Stieltjes integral [7, Theorem 5.4], establishes the relation between Stieltjes derivatives and the Lebesgue-Stieltjes integral for a particularly interesting set of functions.

Theorem 2.1 (Fundamental Theorem of Calculus for the Lebesgue-Stieltjes integral) Let $a, b \in \mathbb{R}, a<b$, and $F:[a, b] \longrightarrow \mathbb{R}$. The following conditions are equivalent.

(1) The function $F$ is absolutely continuous on $[a, b]$ with respect to $g$ (also expressed as $g$-absolutely continuous on $[a, b]$ or $\left.F \in \mathcal{A C}_{g}([a, b])\right)$ according to the following definition: to each $\varepsilon>0$, there is some $\delta>0$ such that, for any family $\left\{\left(a_{n}, b_{n}\right)\right\}_{n=1}^{m}$ of pairwise disjoint open subintervals of $[a, b]$, the inequality

$$
\sum_{n=1}^{m}\left(g\left(b_{n}\right)-g\left(a_{n}\right)\right)<\delta
$$

implies

$$
\sum_{n=1}^{m}\left|F\left(b_{n}\right)-F\left(a_{n}\right)\right|<\varepsilon
$$


(2) The function $F$ fulfills the following properties:

(a) There exists $F_{g}^{\prime}(t)$ for $g$-almost all $t \in[a, b)$ (i.e., for all t except on a set of $\mu_{g}$ measure zero);

(b) $F_{g}^{\prime} \in \mathcal{L}_{g}^{1}([a, b))$, the set of Lebesgue-Stieltjes integrable functions with respect to $\mu_{g} ;$ and

(c) For each $t \in[a, b]$, we have

$$
F(t)=F(a)+\int_{[a, t)} F_{g}^{\prime}(s) d \mu_{g}
$$

In this paper we consider integration in the Lebesgue-Stieltjes sense mainly, and we shall call " $g$-measurable" any function (or set) which is measurable with respect to the Lebesgue-Stieltjes $\sigma$-algebra generated by $g$. Moreover, integrals such as that in (2.1) shall be denoted also as

$$
\int_{[a, t)} F_{g}^{\prime}(s) d g(s)
$$

For properties of $g$-absolutely continuous functions, we refer the readers to [2, 7]. One of the main properties is that every $g$-absolutely continuous function is also $g$-continuous in the sense of the following definition.

Definition 2.2 ([7, Definition 3.1]) A function $F:[a, b] \subset R \rightarrow \mathbb{R}$ is $g$-continuous at $s \in$ $[a, b]$ if, for every $\varepsilon>0$, there exists $\delta>0$ such that

$$
t \in A, \quad|g(t)-g(s)|<\delta \quad \Longrightarrow \quad|f(t)-f(s)|<\varepsilon .
$$

We say that $f$ is $g$-continuous on $A$ if it is $g$-continuous at every point $t_{0} \in[a, b]$.

We shall denote by $\mathcal{B C}_{g}([a, b])$ the set of all $g$-continuous functions that are also bounded. It is shown in [7, Definition 5.5] that $\mathcal{A C}_{g}([a, b])$ is a subset of this set. Hence, the next result gives, indirectly, some properties of $g$-absolutely continuous functions.

Proposition 2.3 ([7, Definition 3.2]) If $F:[a, b] \rightarrow \mathbb{R}$ is a g-continuous function on $[a, b]$, then

(1) $F$ is continuous from the left at every $s \in(a, b]$;

(2) if $g$ is continuous at $s \in[a, b)$, then so is $F$;

(3) if $g$ is constant on some $[c, d] \subset[a, b]$, then so is $F$.

Further properties about the behaviour of $g$-absolutely continuous functions can be found in another result from the same paper.

Proposition 2.4 ([7, Proposition 5.2]) If $F$ is g-absolutely continuous on $[a, b]$, then it has bounded variation.

For the purpose of this paper, we shall also recall the following result. 
Proposition 2.5 ([2, Proposition 5.6]) Let $\mathcal{S} \subset \mathcal{A C}_{g}(I)$ be such that $\left\{F\left(t_{0}\right): F \in \mathcal{S}\right\}$ is bounded. Assume that there exists $h \in \mathcal{L}_{g}^{1}\left(\left[t_{0}, t_{1}\right)\right)$ such that

$$
\left|F_{g}^{\prime}(t)\right| \leq h(t) \quad \text { for g-almost all } t \in\left[t_{0}, t_{1}\right) \text {, and for all } F \in \mathcal{S} \text {. }
$$

Then $\mathcal{S}$ is relatively compact in $\mathcal{B C}_{g}(I)$.

As a final note for this section, we establish the definition of a solution of (1.1), as well as other relevant definitions such as lower and upper solutions.

Definition 2.6 A function $x: I \longrightarrow \mathbb{R}$ is a solution of (1.1) if $x \in \mathcal{A C}_{g}(I), x(0)=0$ and

$$
x_{g}^{\prime}(t)=f(t, x(t)), \quad g \text {-a.a. } t \in I .
$$

We say that $x_{\min }$ is the minimal solution if $x_{\min }$ is a solution and $x_{\min } \leq x$ on $I$ for any other solution $x$. The maximal solution is defined in an analogous way with the obvious changes.

When both the minimal and the maximal solutions exist, we call them the extremal solutions.

Definition 2.7 A function $u: I \longrightarrow \mathbb{R}$ is an upper solution of (1.1) if $u \in \mathcal{A C}_{g}(I), u(0) \geq 0$ and

$$
u_{g}^{\prime}(t) \geq f(t, u(t)), \quad g \text {-a.a. } t \in I
$$

A function $l: I \longrightarrow \mathbb{R}$ is a lower solution of $(1.1)$ if $l \in \mathcal{A C}_{g}(I), l(0) \leq 0$ and

$$
l_{g}^{\prime}(t) \leq f(t, l(t)), \quad g \text {-a.a. } t \in I .
$$

\section{Properties of the infimum of upper solutions}

Consider problem (1.1). We will assume that $f$ satisfies the following hypothesis:

(H1) There exists $M \in \mathcal{L}_{g}^{1}(I)$ such that $|f(t, x)| \leq M(t)$ for $g$-a.a. $t \in I$, all $x \in \mathbb{R}$.

Remark 3.1 If $f$ satisfies a local boundedness condition, such as

$\left(\mathrm{H} 1^{*}\right)$ For each $R>0$, there exists $M_{R} \in \mathcal{L}_{g}^{1}(I)$ such that $|f(t, x)| \leq M_{R}(t)$ for $g$-a.a. $t \in I$, all $x \in \mathbb{R},|x| \leq R$,

we can study the existence of local solutions. To do so, we fix $R>0$, we define

$$
\tilde{f}(t, x)=f(t, \max \{-R, \min \{x, R\}\}),
$$

and we study (1.1) with $f$ replaced by $\tilde{f}$, which satisfies (H1). Observe that solutions of the new problem are local solutions of the former one.

In the following, we shall denote the set of admissible upper solutions for (1.1) as follows:

$$
\mathcal{U}=\left\{u \in \mathcal{A C}_{g}(I): u(0) \geq 0, u_{g}^{\prime}(t) \geq f(t, u(t)) g \text {-a.e. on } I,\left|u_{g}^{\prime}(t)\right| \leq M(t) g \text {-a.e. on } I\right\},
$$


and define $u_{\text {inf }}(t):=\inf \{u(t): u \in \mathcal{U}\}, t \in I$. Note that $u_{\text {inf }}(0)=0$ as the function $u$ given by $u(t)=\int_{[0, t)} M(s) d g(s)$ belongs to $\mathcal{U}$ and, trivially, $u(0)=0$.

Since the aim of this paper is to find out some conditions guaranteeing that the function $u_{\mathrm{inf}}$ is the minimal solution of the problem, we first need to obtain conditions that assure that $u_{\text {inf }} \in \mathcal{A C}_{g}(I)$ and $\left|\left(u_{\text {inf }}\right)_{g}^{\prime}\right| \leq M$. In order to do so, we need the following lemma, in which the first condition for our goal, due to Antunes Monteiro and Slavík (see condition (C4) in [1]), will appear.

Lemma 3.2 Consider $\beta_{1}, \beta_{2}, \ldots, \beta_{n} \in \mathcal{U}$. Iff verifies $(\mathrm{H} 1)$ and

(H2) For all $t \in I \cap D_{g}$, the map $u \in \mathbb{R} \mapsto u+f(t, u)\left(g\left(t^{+}\right)-g(t)\right)$ is nondecreasing, then the function $\beta_{\min }(t)=\min \left\{\beta_{1}(t), \beta_{2}(t), \ldots, \beta_{n}(t)\right\}, t \in I$, is an element of $\mathcal{U}$.

Proof To prove this result, it suffices to show that given $\beta_{1}, \beta_{2} \in \mathcal{U}, \beta_{\min }(t)=\min \left\{\beta_{1}(t)\right.$, $\left.\beta_{2}(t)\right\}, t \in I$, belongs to $\mathcal{U}$. First of all, note that $\beta_{\min } \in \mathcal{A C}_{g}(I)$ since we can write it as the difference of two $g$-absolutely continuous functions:

$$
\beta_{\min }(t)=\frac{\beta_{1}(t)-\beta_{2}(t)}{2}-\frac{\left|\beta_{1}(t)-\beta_{2}(t)\right|}{2} .
$$

Moreover, $\beta_{\min }(0) \geq 0$ trivially, and so, all that is left to prove is that for $g$-a.a. $t \in I$ $\left(\beta_{\text {min }}\right)_{g}^{\prime}(t) \geq f\left(t, \beta_{\text {min }}(t)\right)$ and $\left|\left(\beta_{\min }\right)_{g}^{\prime}(t)\right| \leq M(t)$.

Let $E=\left\{t \in I: \exists\left(\beta_{1}\right)_{g}^{\prime}(t),\left(\beta_{2}\right)_{g}^{\prime}(t),\left(\beta_{\min }\right)_{g}^{\prime}(t)\right\}$, and let $t_{0} \in E$. Note that $t_{0} \notin C_{g}$ since there exist $g$-derivatives at that point. We distinguish two possible cases: either $\beta_{1} \geq \beta_{2}$ on a set $S \subset[0,1]$ such that $t_{0} \in\left[S \cap\left(t_{0}, 1\right)\right]^{\prime}$ or $\beta_{1}<\beta_{2}$ on $\left(t_{0}, t_{0}+\delta\right)$ for some $\delta>0$. Assume the first one holds. If $\beta_{1}\left(t_{0}\right) \geq \beta_{2}\left(t_{0}\right)$, then

$$
\begin{aligned}
\left(\beta_{\min }\right)_{g}^{\prime}\left(t_{0}\right) & =\lim _{t \rightarrow t_{0}^{+}} \frac{\beta_{\min }(t)-\beta_{\min }\left(t_{0}\right)}{g(t)-g\left(t_{0}\right)}=\lim _{t \rightarrow t_{0}^{+}, t \in S \cap\left(t_{0}, 1\right)} \frac{\beta_{\min }(t)-\beta_{\min }\left(t_{0}\right)}{g(t)-g\left(t_{0}\right)} \\
& =\lim _{t \rightarrow t_{0}^{+}} \frac{\beta_{2}(t)-\beta_{2}\left(t_{0}\right)}{g(t)-g\left(t_{0}\right)}=\left(\beta_{2}\right)_{g}^{\prime}\left(t_{0}\right) \geq f\left(t_{0}, \beta_{2}\left(t_{0}\right)\right)=f\left(t_{0}, \beta_{\min }\left(t_{0}\right)\right) .
\end{aligned}
$$

Otherwise, $\beta_{2}\left(t_{0}\right)>\beta_{1}\left(t_{0}\right)$, and so $t_{0} \in D_{g}$. Note that $\beta_{1}\left(t_{0}^{+}\right)=\lim _{t \rightarrow t_{0}^{+}, t \in S \cap\left(t_{0}, 1\right)} \beta_{1}(t) \geq \beta_{2}\left(t_{0}^{+}\right)$. Hence, using hypothesis (H2),

$$
\begin{aligned}
\left(\beta_{\min }\right)_{g}^{\prime}\left(t_{0}\right) & =\frac{\beta_{\min }\left(t_{0}^{+}\right)-\beta_{\min }\left(t_{0}\right)}{g\left(t_{0}^{+}\right)-g\left(t_{0}\right)}=\frac{\beta_{2}\left(t_{0}^{+}\right)-\beta_{1}\left(t_{0}\right)}{g\left(t_{0}^{+}\right)-g\left(t_{0}\right)}=\frac{\beta_{2}\left(t_{0}\right)+\Delta g\left(t_{0}\right)\left(\beta_{2}\right)_{g}^{\prime}-\beta_{1}\left(t_{0}\right)}{\Delta g\left(t_{0}\right)} \\
& \geq \frac{\beta_{2}\left(t_{0}\right)+\Delta g\left(t_{0}\right) f\left(t_{0}, \beta_{2}\left(t_{0}\right)\right)-\beta_{1}\left(t_{0}\right)}{\Delta g\left(t_{0}\right)} \geq \frac{\beta_{1}\left(t_{0}\right)+\Delta g\left(t_{0}\right) f\left(t_{0}, \beta_{1}\left(t_{0}\right)\right)-\beta_{1}\left(t_{0}\right)}{\Delta g\left(t_{0}\right)} \\
& =f\left(t_{0}, \beta_{1}\left(t_{0}\right)\right)=f\left(t_{0}, \beta_{\min }\left(t_{0}\right)\right) .
\end{aligned}
$$

Thus $\left(\beta_{\min }\right)_{g}^{\prime}(t) \geq f\left(t, \beta_{\min }(t)\right)$ for $g$-a.a. $t \in I$. Moreover, $\left.\mid\left(\beta_{\min }\right)_{g}^{\prime}\right) \mid \leq M$. Indeed, if $\beta_{1}\left(t_{0}\right) \geq$ $\beta_{2}\left(t_{0}\right)$, then it is clear. If $\beta_{1}\left(t_{0}\right)<\beta_{2}\left(t_{0}\right)$, we have $\left(\beta_{\min }\right)_{g}^{\prime}\left(t_{0}\right) \geq f\left(t_{0}, \beta_{\min }\left(t_{0}\right)\right) \geq-M\left(t_{0}\right)$ and

$$
\left(\beta_{\min }\right)_{g}^{\prime}\left(t_{0}\right)=\frac{\beta_{2}\left(t_{0}^{+}\right)-\beta_{1}\left(t_{0}\right)}{\Delta g\left(t_{0}\right)} \leq \frac{\beta_{1}\left(t_{0}^{+}\right)-\beta_{1}\left(t_{0}\right)}{\Delta g\left(t_{0}\right)}=\left(\beta_{1}\right)_{g}^{\prime}\left(t_{0}\right) \leq M\left(t_{0}\right) .
$$

The case $\beta_{1}<\beta_{2}$ on $\left(t_{0}, t_{0}+\delta\right)$ for some $\delta>0$ is similar. 
Using the previous lemma, one can show that $u_{\text {inf }}$ verifies some of the required properties.

Lemma 3.3 Iff satisfies hypotheses $(\mathrm{H} 1)-(\mathrm{H} 2)$, then $u_{\mathrm{inf}} \in \mathcal{A C}_{g}(I)$ and

$$
\left|\left(u_{\text {inf }}\right)_{g}^{\prime}(t)\right| \leq M(t), \quad \text { g-a.a. } t \in I \text {. }
$$

Proof Let $s, t \in I$ be such that $s<t$. By definition of $u_{\text {inf }}$, given $\varepsilon>0$, there exist $u_{1}, u_{2} \in \mathcal{U}$ such that

$$
0 \leq u_{1}(t)-u_{\text {inf }}(t)<\frac{\varepsilon}{2}, \quad 0 \leq u_{2}(s)-u_{\text {inf }}(s)<\frac{\varepsilon}{2} .
$$

Define $u(z)=\min \left\{u_{1}(z), u_{2}(z)\right\}$ for all $z \in I$. By Lemma 3.2, $u \in \mathcal{U}$. Moreover, $0 \leq u(t)-$ $u_{\text {inf }}(t)<\varepsilon / 2,0 \leq u(s)-u_{\text {inf }}(s)<\varepsilon / 2$. Hence,

$$
\begin{aligned}
\left|u_{\text {inf }}(t)-u_{\text {inf }}(s)\right| & \leq\left|u_{\text {inf }}(t)-u(t)\right|+|u(t)-u(s)|+\left|u(s)-u_{\text {inf }}(s)\right| \\
& <\frac{\varepsilon}{2}+\left|\int_{[s, t)} M d g\right|+\frac{\varepsilon}{2}=\int_{[s, t)} M d g+\varepsilon .
\end{aligned}
$$

Since $\varepsilon>0$ is arbitrary, we have that $\left|u_{\text {inf }}(t)-u_{\text {inf }}(s)\right| \leq \int_{[s, t)} M d g$. Now, using that $M \in$ $\mathcal{L}_{g}^{1}(I)$, it is easy to check using standard arguments that $u_{\text {inf }} \in \mathcal{A C}_{g}(I)$. Moreover, for each $s \in I$ that $\left(u_{\text {inf }}\right)_{g}^{\prime}(s)$ exists, define $\Phi_{s}(t)=\int_{[s, t)} M d g, t \in I, t>s$. Note that $\Phi_{s}$ is $g$-absolutely continuous, so, by the fundamental theorem of calculus [7, Theorem 2.4], we have that

$$
\left|\left(u_{\mathrm{inf}}\right)_{g}^{\prime}(t)\right|=\lim _{t \rightarrow s^{+}} \frac{\left|u_{\mathrm{inf}}(t)-u_{\mathrm{inf}}(s)\right|}{g(t)-g(s)} \leq \lim _{t \rightarrow s^{+}} \frac{\Phi_{s}(t)-\Phi_{s}(s)}{g(t)-g(s)}=\left(\Phi_{s}\right)_{g}^{\prime}(s)=M(s) .
$$

Now, since $u_{\text {inf }} \in \mathcal{A C}_{g}(I)$, we have that $\left(u_{\text {inf }}\right)_{g}^{\prime}(s)$ exists for $g$-a.a. $s \in I$, and the result follows.

Furthermore, one can show that $u_{\text {inf }}$ can be approximated by a sequence of $\mathcal{U}$.

Lemma 3.4 Iff verifies (H1)-(H2), then there exists a nonincreasing sequence $\left\{u_{n}\right\} \subset \mathcal{U}$ that converges uniformly on I to $u_{\mathrm{inf}}$.

Proof For each $t \in[0,1]$, define $u_{0}(t)=\int_{[0, t)} M(\tau) d g(\tau) \in \mathcal{U}$. Assume that $u_{1}, u_{2}, \ldots, u_{n-1} \in$ $\mathcal{U}$ have been defined. For every $i \in\{0,1, \ldots, n-1\}$, choose $y_{i} \in \mathcal{U}$ satisfying the following inequalities:

$$
u_{\text {inf }}\left(\frac{i}{n}\right) \leq y_{i}\left(\frac{i}{n}\right) \leq u_{\text {inf }}\left(\frac{i}{n}\right)+\frac{1}{n} .
$$

Define $u_{n}=\min \left\{u_{n-1}, y_{0}, \ldots, y_{n-1}\right\}$. Then $u_{n} \in \mathcal{U}$ by Lemma 3.2; moreover, the sequence $\left\{u_{n}\right\}_{n=1}^{\infty}$ is nonincreasing. Furthermore, $\left\{u_{n}\right\}_{n=1}^{\infty}$ verifies Proposition 2.5 since, for each $n \in \mathbb{N},\left|\left(u_{n}\right)_{g}^{\prime}(t)\right| \leq M(t)$ for $g$-a.a. $t \in I$ and $\left\{u_{n}(0): n \in \mathbb{N}\right\}=\{0\}$ as $0 \leq u_{n}(0) \leq u_{1}(0)=0$. Hence, $\left\{u_{n}\right\}$ is a relatively compact set, and therefore there exists a subsequence $\left\{u_{n_{k}}\right\}$ that converges uniformly in $\mathcal{B C}_{g}(I)$ to a limit, say $v$. Since $\left\{u_{n}\right\}$ is a monotone sequence, it also converges uniformly to $v$. Therefore, it is enough to show that $v=u_{\text {inf }}$. 
Since $u_{n} \geq u_{\text {inf }}$ for all $n \in \mathbb{N}$, we have that $v \geq u_{\text {inf. }}$. Assume that $v \neq u_{\text {inf. }}$. Then there exists $t_{0} \in I$ such that $v\left(t_{0}\right)>u_{\text {inf }}\left(t_{0}\right)$. Both functions belong to $\mathcal{B C}_{g}(I)$, so Proposition 2.3 ensures that they are left-continuous. Hence, there exist $c>0$ and $\delta>0$ such that $u_{\text {inf }}(t)<v(t)-c$ for all $t \in\left(t_{0}-\delta, t_{0}\right]$. Consider $n \in \mathbb{N}$ such that $1 / n<\min \{c, \delta\}$. Then $u_{\text {inf }}(t)<v(t)-c \leq$ $u_{n}(t)-c \leq u_{n}(t)-1 / n$ for all $t \in\left(t_{0}-\delta, t_{0}\right]$, and so $u_{\text {inf }}(t)+1 / n<u_{n}(t)$ for all $t \in\left(t_{0}-1 / n, t_{0}\right]$. Now, for some $i=0,1, \ldots, n, i / n \in\left(t_{0}-1 / n, t_{0}\right]$, and so $u_{\text {inf }}(i / n)+1 / n<u_{n}(i / n)$, which is a contradiction. Therefore, $v=u_{\text {inf }}$.

In the last two theorems of this section, we study the behaviour of $f$ over the graph of $u_{\text {inf }}$, from which one can obtain conditions over $f$ so that $u_{\text {inf }}$ is a solution.

Theorem 3.5 Consider (1.1) under hypotheses (H1)-(H2). Then, for g-a.a.t $\in I$,

$$
\left(u_{\text {inf }}\right)_{g}^{\prime}(t) \geq f\left(t, u_{\text {inf }}(t)\right) \chi_{I_{1}}(t)+\liminf _{y \rightarrow\left(u_{\text {inf }}(t)\right)^{+}} f(t, y) \chi_{I_{2}}(t),
$$

where $I_{1}=\left\{t \in I: u_{\text {inf }}(t)=u(t), u_{g}^{\prime}(t) \geq f(t, u(t))\right.$ for some $\left.u \in \mathcal{U}\right\} \cup D_{g}$ and $I_{2}=I \backslash I_{1}$.

Proof First, note that hypotheses $(\mathrm{H} 1)-(\mathrm{H} 2)$ guarantee that $u_{\text {inf }} \in \mathcal{A C}_{g}(I)$, and therefore $\left(u_{\text {inf }}\right)_{g}^{\prime}$ exists $g$-almost everywhere.

Let $s \in I_{1} \backslash D_{g}$ be such that $\left(u_{\text {inf }}\right)_{g}^{\prime}(s)$ exists, and let $u \in \mathcal{U}$ be the corresponding function to the definition of $I_{1}$. Then $\left(u_{\text {inf }}\right)_{g}^{\prime}(s)=u_{g}^{\prime}(s) \geq f(t, u(s))=f\left(t, u_{\text {inf }}(s)\right)$. On the other hand, for $s \in D_{g}$, consider a sequence $\left\{u_{n}\right\}_{n=1}^{\infty} \subset \mathcal{U}$ as in Lemma 3.4. We know that, for all $n$, it holds that

$$
u_{n}\left(s^{+}\right) \geq u_{n}(s)+\Delta g(s) f\left(s, u_{n}(s)\right) \geq u_{\mathrm{inf}}(s)+\Delta g(s) f\left(s, u_{\mathrm{inf}}(s)\right)
$$

Hence, since $\left\{u_{n}\right\}$ converges uniformly to $u_{\text {inf }}$, it follows from the Moore-Osgood theorem [3, Chapter VII, Theorem 2] that

$$
u_{\text {inf }}\left(s^{+}\right) \geq u_{\text {inf }}(s)+\Delta g(s) f\left(s, u_{\text {inf }}(s)\right)
$$

or equivalently, $\left(u_{\text {inf }}\right)_{g}^{\prime}(s) \geq f\left(s, u_{\text {inf }}(s)\right)$.

Finally, we study $\left(u_{\text {inf }}\right)_{g}^{\prime}$ on $I_{2}$. To do so, we consider again a sequence $\left\{u_{n}\right\}_{n=1}^{\infty} \subset \mathcal{U}$ as in Lemma 3.4. Since $\left|\left(u_{n}\right)_{g}^{\prime}\right|$ is uniformly $\mathcal{L}_{g}^{1}$-bounded on $I$, we have that $\liminf _{n \rightarrow \infty}\left(u_{n}\right)_{g}^{\prime} \in$ $\mathcal{L}_{g}^{1}(I)$. Moreover, by Fatou's lemma, for $\tilde{t}<t$,

$$
u_{\mathrm{inf}}(t)-u_{\mathrm{inf}}(\tilde{t})=\liminf _{n \rightarrow \infty}\left(u_{n}(t)-u_{n}(\tilde{t})\right)=\liminf _{n \rightarrow \infty} \int_{[\tilde{t}, t)}\left(u_{n}\right)_{g}^{\prime} d g \geq \int_{[\tilde{t}, t)} \liminf _{n \rightarrow \infty}\left(u_{n}\right)_{g}^{\prime} d g
$$

Now, since $u_{\text {inf }} \in \mathcal{A C}_{g}(I)$, we have that $u_{\text {inf }}(t)-u_{\text {inf }}(\tilde{t})=\int_{[\tilde{t}, t)}\left(u_{\text {inf }}\right)_{g}^{\prime} d g$. Hence,

$$
\left(u_{\text {inf }}\right)_{g}^{\prime}(t) \geq \liminf _{n \rightarrow \infty}\left(u_{n}\right)_{g}^{\prime}(t) \geq \liminf _{n \rightarrow \infty} f\left(t, u_{n}(t)\right) \quad g \text {-a.a. } t \in I .
$$

Now, if $s \in I_{2}$ and $u_{\text {inf }}(s)=u_{n}(s)$ for some $n$, the definition of $I_{2}$ implies that $s \notin D_{g}$ and either $\left(u_{n}\right)_{g}^{\prime}(s)$ does not exist or $\left(u_{n}\right)_{g}^{\prime}(s)<f\left(s, u_{n}(s)\right)$. The set

$$
\bigcup_{n \in \mathbb{N}}\left(\left\{t \in I \backslash D_{g}: \nexists\left(u_{n}\right)_{g}^{\prime}(s)\right\} \cup\left\{t \in I \backslash D_{g}:\left(u_{n}\right)_{g}^{\prime}(s)<f\left(s, u_{n}(s)\right)\right\}\right)
$$


is a null-measure set with respect to the $g$-measure. Hence, for $g$-a.a. $t \in I_{2}$, we have that $u_{\text {inf }}(t)<u_{n}(t)$ for all $n \in \mathbb{N}$, and so, since $\left\{u_{n}(t)\right\}$ is one of the infinitely many sequences that converge to $u_{\text {inf }}(t)^{+}$, we have that

$$
\left(u_{\text {inf }}\right)_{g}^{\prime}(t) \geq \liminf _{n \rightarrow \infty} f\left(t, u_{n}(t)\right) \geq \liminf _{y \rightarrow\left(u_{\text {inf }}(t)\right)^{+}} f(t, y),
$$

which concludes the proof.

Remark 3.6 It follows from Theorem 3.5 that if the following condition is satisfied

$$
\liminf _{y \rightarrow\left(u_{\text {inf }}(t)\right)^{+}} f(t, y) \geq f\left(t, u_{\text {inf }}(t)\right), \quad \text { for } g \text {-a.a. } t \in I,
$$

then $\left(u_{\text {inf }}\right)_{g}^{\prime} \geq f\left(t, u_{\text {inf }}(t)\right)$, i.e., $u_{\text {inf }}$ is an upper solution.

Note, however, that for all $t \in I \cap D_{g}, u_{\text {inf }}$ is a "solution", i.e., $\left(u_{\text {inf }}(t)\right)_{g}^{\prime}=f\left(t, u_{\text {inf }}(t)\right)$ as long as hypotheses $(\mathrm{H} 1)-(\mathrm{H} 2)$ are satisfied. Indeed, we already know that $\left(u_{\mathrm{inf}}\right)_{g}^{\prime}(t) \geq f\left(t, u_{\mathrm{inf}}(t)\right)$ for $t \in I \cap D_{g}$. Reasoning by contradiction, assume that there exists $t_{0} \in I \cap D$ such that $\left(u_{\text {inf }}\right)_{g}^{\prime}\left(t_{0}\right)>f\left(t_{0}, u_{\text {inf }}\left(t_{0}\right)\right)$, or equivalently, $u_{\text {inf }}\left(t_{0}^{+}\right)>u_{\text {inf }}\left(t_{0}\right)+\Delta g\left(t_{0}\right) f\left(t_{0}, u_{\text {inf }}\left(t_{0}\right)\right)=a$. Then one can find $z_{0} \in\left(a, u_{\text {inf }}\left(t_{0}^{+}\right)\right)$. Define

$$
u(t)= \begin{cases}u_{\mathrm{inf}}(t) & \text { if } t \in\left[0, t_{0}\right] \\ z_{0}+\int_{\left(t_{0}, t\right)} M(\tau) d g(\tau) & \text { if } t \in\left(t_{0}, 1\right]\end{cases}
$$

First, note that

$$
u_{g}^{\prime}\left(t_{0}\right)=\frac{u\left(t_{0}^{+}\right)-u\left(t_{0}\right)}{\Delta g\left(t_{0}\right)}=\frac{z_{0}-u_{\mathrm{inf}}\left(t_{0}\right)}{\Delta g\left(t_{0}\right)}>\frac{a-u_{\mathrm{inf}}\left(t_{0}\right)}{\Delta g\left(t_{0}\right)}=f\left(t_{0}, u_{\mathrm{inf}}\left(t_{0}\right)\right)=f\left(t_{0}, u\left(t_{0}\right)\right) .
$$

Moreover, $\left|u_{g}^{\prime}\right| \leq M$ trivially and $u \in \mathcal{A} C_{g}(I)$ as it is defined as a piecewise function of $\mathcal{A C}_{g}(I)$ functions. Hence, $u \in \mathcal{U}$, which is a contradiction, as $u\left(t_{0}^{+}\right)=z_{0}<u_{\text {inf }}\left(t_{0}^{+}\right)$.

Therefore, in order to determine the conditions guaranteeing that $u_{\text {inf }}$ is a solution, there is no need to see what happens at points of $D_{g}$ as long as (H1)-(H2) hold.

We now prove the following lemma that we will need in order to obtain a necessary condition for $u_{\text {inf }}$ being an upper solution.

Lemma 3.7 Let $M:[0,1] \rightarrow[0, \infty]$ be a g-integrable function. If $F \subset[0,1]$ is a set of positive g-measures, then there exists $F_{1} \subset F$ such that, for all $s \in F_{1}$,

$$
\lim _{t \rightarrow s^{+}} \frac{g(t)-g(s)}{\mu_{g}([s, t) \cap F)}=1, \quad \lim _{t \rightarrow s^{+}} \frac{1}{\mu_{g}([s, t) \cap F)} \int_{[s, t) \backslash F} M(\tau) d g(\tau)=0 .
$$

Proof First, let $G: I=[0,1] \rightarrow \mathbb{R}$ be the map given by

$$
G(0)=0, \quad G(t)=\int_{[0, t)} \chi_{F}(s) d g(s), \quad \forall t \in(0,1],
$$

where $\chi_{F}$ denotes the characteristic function of the set $F$. Clearly $\chi_{F} \in \mathcal{L}_{g}^{1}((0,1])$ and therefore it is trivial that $G \in \mathcal{A C}_{g}(I)$. Hence, there exists a set $F_{0} \subset F$ such that $\mu_{g}\left(F \backslash F_{0}\right)=0$ 
and there exists $G_{g}^{\prime}(s)$ for all $s \in F_{0}$. Moreover, $G_{g}^{\prime}(s)=\chi_{F}(s)=1$ for all $s \in F_{0}$. Thus,

$$
\begin{aligned}
1 & =G_{g}^{\prime}(s)=\lim _{t \rightarrow s^{+}} \frac{G(t)-G(s)}{g(t)-g(s)}=\lim _{t \rightarrow s^{+}} \frac{\int_{[0, t)} \chi_{F}(\tau) d g(\tau)-\int_{[0, s)} \chi_{F}(\tau) d g(\tau)}{g(t)-g(s)} \\
& =\lim _{t \rightarrow s^{+}} \frac{\int_{[s, t) \cap F} d g(\tau)}{g(t)-g(s)}=\lim _{t \rightarrow s^{+}} \frac{\mu_{g}([s, t) \cap F)}{g(t)-g(s)} .
\end{aligned}
$$

Consider now the map $H: I \rightarrow \mathbb{R}$ defined as

$$
H(0)=0, \quad H(t)=\int_{[0, t)} M(s) \cdot \chi_{I \backslash F} d g(s), \quad \forall t \in(0,1] .
$$

Once again, since $M_{0}=M \cdot \chi_{I \backslash F} \in \mathcal{L}_{g}^{1}((0,1])$, it follows that $H \in \mathcal{A C}_{g}(I)$, and therefore there exists $F_{1} \subset F_{0}$ such that $\mu_{g}\left(F_{0} \backslash F_{1}\right)=0$ and $H_{g}^{\prime}(s)$ exists for all $s \in F_{1}$. Moreover, $H_{g}^{\prime}(s)=M(s) \cdot \chi_{I \backslash F}(s)=0$ for all $s \in F_{1}$. Hence,

$$
0=H_{g}^{\prime}(s)=\lim _{t \rightarrow s^{+}} \frac{H(t)-H(s)}{g(t)-g(s)}=\lim _{t \rightarrow s^{+}} \frac{\int_{[0, t)} M_{0}(\tau) d g(\tau)-\int_{[0, s)} M_{0}(\tau) d g(\tau)}{g(t)-g(s)} .
$$

Now, since $s \in F_{1} \subset F_{0}$, we have that

$$
\begin{aligned}
0 & =\lim _{t \rightarrow s^{+}} \frac{\int_{[s, t)} M_{0}(\tau) d g(\tau)}{g(t)-g(s)}=\lim _{t \rightarrow s^{+}} \frac{\int_{[s, t)} M_{0}(\tau) d g(\tau)}{g(t)-g(s)} \cdot \lim _{t \rightarrow s^{+}} \frac{g(t)-g(s)}{\mu_{g}([s, t) \cap F)} \\
& =\lim _{t \rightarrow s^{+}} \frac{\int_{[s, t)} M_{0}(\tau) d g(\tau)}{g(t)-g(s)} \cdot \frac{g(t)-g(s)}{\mu_{g}([s, t) \cap F)}=\lim _{t \rightarrow s^{+}} \frac{\int_{[s, t)} M_{0}(\tau) d g(\tau)}{\mu_{g}([s, t) \cap F)},
\end{aligned}
$$

and so, for all $s \in F_{1}$, we have

$$
\lim _{t \rightarrow s^{+}} \frac{g(t)-g(s)}{\mu_{g}([s, t) \cap F)}=1, \quad \lim _{t \rightarrow s^{+}} \frac{1}{\mu_{g}([s, t) \cap F)} \int_{[s, t) \backslash F} M(\tau) d g(\tau)=0 .
$$

We can now state and prove the following necessary condition for $u_{\text {inf }}$ being an upper solution.

Theorem 3.8 Consider problem (1.1) under hypotheses $(\mathrm{H} 1)-(\mathrm{H} 2)$. Assume $\left(u_{\mathrm{inf}}\right)_{g}^{\prime}(t) \geq$ $f\left(t, u_{\text {inf }}(t)\right)$ for $g$-a.a. $t \in I$. Then:

(a) The set $J=\left\{t \in I \backslash D_{g}:\left(u_{\text {inf }}\right)_{g}^{\prime}(t)>\lim \sup _{y \rightarrow\left(u_{\text {inf }}(t)\right)^{-}} f(t, y)\right\}$ is a countable union of sets which contain no positive measure set. Specifically, $J=\bigcup_{n, m \in \mathbb{N}} J_{n, m}$, where

$$
J_{n, m}=\left\{t \in I \backslash D_{g}:\left(u_{\mathrm{inf}}\right)_{g}^{\prime}(t)-\frac{1}{n}>\sup \left\{f(t, y): u_{\mathrm{inf}}(t)-\frac{1}{m}<y<u_{\mathrm{inf}}(t)\right\}\right\} .
$$

(b) $\left(u_{\text {inf }}\right)_{g}^{\prime}(t) \leq \lim \sup _{y \rightarrow\left(u_{\text {inf }}(t)\right)^{-}} f(t, y)$ for $g$-a.a. $t \in I \backslash D_{g}$ provided that, for all $n, m \in \mathbb{N}$, the set $J_{n, m}$ is $g$-measurable.

Proof For each $t \in J$, there exists $n \in \mathbb{N}$ such that

$$
\left(u_{\text {inf }}\right)_{g}^{\prime}(t)-\frac{1}{n}>\limsup _{y \rightarrow\left(u_{\text {inf }}(t)\right)^{-}} f(t, y)=\inf _{\varepsilon>0}\left\{\sup _{u_{\text {inf }}(t)-\varepsilon<y<u_{\text {inf }}(t)} f(t, y)\right\} .
$$


Therefore, there exists $m \in \mathbb{N}$ such that $\left(u_{\text {inf }}\right)_{g}^{\prime}(t)-1 / n>\sup \left\{f(t, y): u_{\text {inf }}-1 / m<y<u_{\text {inf }}(t)\right\}$, and so $t \in J_{n, m}$. Conversely, if $t \in J_{n, m}$ for some $n, m \in \mathbb{N}$, then

$$
\left(u_{\text {inf }}\right)_{g}^{\prime}(t)-\frac{1}{n}>\sup _{u_{\text {inf }}(t)-1 / m<y<u_{\text {inf }}} f(t, y) \geq \limsup _{y \rightarrow\left(u_{\text {inf }}(t)\right)^{-}} f(t, y) .
$$

Hence, $t \in J$ and we can write $J=\bigcup_{n, m \in \mathbb{N}} J_{n, m}$. Thus, it is enough to show that, for all $n, m \in \mathbb{N}, J_{n, m}$ contains no positive $g$-measure subset.

Reasoning by contradiction, assume that there exist $n, m \in \mathbb{N}$ such that $J_{n, m}$ contains a subset of positive $g$-measure, denoted again by $J_{n, m}$ for simplicity. By Lemma 3.7 there exist $t_{0} \in J_{n, m} \cap(0,1)$ and $\delta>0$ such that, for all $t \in\left(t_{0}, t_{0}+\delta\right)$,

$$
\mu_{g}\left(\left[t_{0}, t\right) \cap J_{n, m}\right) \geq \frac{1}{2}\left(g(t)-g\left(t_{0}\right)\right), \quad \int_{\left[t_{0}, t\right) \bigvee_{n, m}} M(s) d g(s) \leq \frac{1}{4 n} \mu_{g}\left(\left[t_{0}, t\right) \cap J_{n, m}\right) .
$$

Moreover, since $t_{0} \notin D_{g}, \delta$ can be chosen so that $g(t)-g\left(t_{0}\right)<n / m$ for all $t \in\left(t_{0}, t_{0}+\delta\right)$. Let us define $u \in \mathcal{A C}_{g}(I)$ such that $u(0)=0$ and, for all $t \in I$,

$$
u_{g}^{\prime}(t)= \begin{cases}\left(u_{\mathrm{inf}}\right)_{g}^{\prime}(t) & \text { if } t<t_{0}, \\ \left(u_{\mathrm{inf}}\right)_{g}^{\prime}(t)-1 / n & \text { if } t \in\left[t_{0}, t_{0}+\delta\right] \cap J_{n, m}, \\ M(t) & \text { otherwise. }\end{cases}
$$

First of all, note that

$$
u\left(t_{0}\right)=\int_{\left[0, t_{0}\right)} u_{g}^{\prime}(s) d g(s)=\int_{\left[0, t_{0}\right)} u_{\mathrm{inf}}^{\prime}(s) d g(s)=u_{\mathrm{inf}}\left(t_{0}\right) .
$$

Moreover, note that $\left|u_{g}^{\prime}(t)\right| \leq M(t)$ for $g$-a.a. $t \in I \backslash\left(\left[t_{0}, t_{0}+\delta\right] \cap J_{n, m}\right)$. For $t \in\left[t_{0}, t_{0}+\delta\right] \cap J_{n, m}$, we have that $\left(u_{\text {inf }}\right)_{g}^{\prime}(t)-1 / n \leq M(t)-1 / n<M(t)$ and

$$
\left(u_{\text {inf }}\right)_{g}^{\prime}(t)-\frac{1}{n}>\sup \left\{f(t, y): u_{\text {inf }}(t)-\frac{1}{m}<y<u_{\text {inf }}(t)\right\} \geq-M(t)
$$

Thus $\left|u_{g}^{\prime}(t)\right| \leq M(t)$ for $g$-a.a. $t \in I$.

Now, since $u_{\text {inf }}$ is an upper solution by hypothesis, it is trivial that $u_{g}^{\prime}(t) \geq f(t, u(t))$ for $g$-a.a. $t \in I \backslash\left(\left[t_{0}, t_{0}+\delta\right] \cap J_{n, m}\right)$. For $t \in\left(t_{0}, t_{0}+\delta\right)$, we have

$$
\begin{aligned}
u_{\text {inf }}(t)-u(t) & =\int_{\left[t_{0}, t\right)}\left(\left(u_{\mathrm{inf}}\right)_{g}^{\prime}(s)-u_{g}^{\prime}(s)\right) d g(s) \\
& =\int_{\left[t_{0}, t\right) \cap \cap_{n, m}}\left(\left(u_{\mathrm{inf}}\right)_{g}^{\prime}(s)-u_{g}^{\prime}(s)\right) d g(s)+\int_{\left[t_{0}, t\right) \bigvee_{n, m}}\left(\left(u_{\mathrm{inf}}\right)_{g}^{\prime}(s)-u_{g}^{\prime}(s)\right) d g(s) \\
& =\int_{\left[t_{0}, t\right) \cap \sum_{n, m}} \frac{1}{n} d g(s)+\int_{\left[t_{0}, t\right) \backslash \bigvee_{n, m}}\left(\left(u_{\mathrm{inf}}\right)_{g}^{\prime}(s)-M(s)\right) d g(s) \\
& =\frac{1}{n} \mu_{g}\left(\left[t_{0}, t\right) \cap J_{n, m}\right)+\int_{\left[t_{0}, t\right) \backslash n, m}\left(\left(u_{\mathrm{inf}}\right)_{g}^{\prime}(s)-M(s)\right) d g(s) .
\end{aligned}
$$

Hence, on the one hand,

$$
u_{\text {inf }}(t)-u(t) \geq \frac{1}{n} \mu_{g}\left(\left[t_{0}, t\right) \cap J_{n, m}\right)-2 \int_{\left[t_{0}, t\right) \bigvee_{n, m}} M(s) d g(s)
$$




$$
\geq \frac{1}{n} \mu_{g}\left(\left[t_{0}, t\right) \cap J_{n, m}\right)-\frac{1}{2 n} \mu_{g}\left(\left[t_{0}, t\right) \cap J_{n, m}\right)=\frac{1}{2 n} \mu_{g}\left(\left[t_{0}, t\right) \cap J_{n, m}\right)>0 .
$$

On the other hand,

$$
\begin{aligned}
u_{\text {inf }}(t)-u(t) & =\frac{1}{n} \mu_{g}\left(\left[t_{0}, t\right) \cap J_{n, m}\right)+\int_{\left[t_{0}, t\right) \backslash n, m}\left(\left(u_{\text {inf }}\right)_{g}^{\prime}(s)-M(s)\right) d g(s) \\
& \leq \frac{1}{n} \mu_{g}\left(\left[t_{0}, t\right) \cap J_{n, m}\right) \leq \frac{1}{n} \mu_{g}\left(\left[t_{0}, t\right)\right)=\frac{1}{n}\left(g(t)-g\left(t_{0}\right)\right)<\frac{1}{m} .
\end{aligned}
$$

That is, for $t \in\left(t_{0}, t_{0}+\delta\right)$, we have $0<u_{\text {inf }}(t)-u(t)<1 / m$, or equivalently $u_{\text {inf }}(t)-1 / m<$ $u(t)<u_{\text {inf }}(t)$. Therefore, for $g$-a.a. $t \in\left(t_{0}, t_{0}+\delta\right) \cap J_{n, m}$, we have

$$
u_{g}^{\prime}(t)=\left(u_{\mathrm{inf}}\right)_{g}^{\prime}(t)-\frac{1}{n}>\sup _{u_{\mathrm{inf}}(t)-1 / m<y<u_{\mathrm{inf}}(t)} f(t, y) \geq f(t, u(t))
$$

Thus, $u_{g}^{\prime}(t) \geq f(t, u(t))$ for $g$-a.a. $t \in I$, i.e., $u \in \mathcal{U}$, which is a contradiction, since $u_{\text {inf }}(t)-$ $u(t)>0$ for all $t \in\left(t_{0}, t_{0}+\delta\right)$.

Part (b) follows from (a) and the extra assumption.

Combining Remark 3.6 with part (b) of Theorem 3.8, it is easy to see that $u_{\mathrm{inf}}$ is a solution of (1.1) if the sets $J_{n, m}$ are $g$-measurable and

$$
\limsup _{y \rightarrow\left(u_{\text {inf }}(t)\right)^{-}} f(t, y) \leq f\left(t, u_{\text {inf }}(t)\right) \leq \liminf _{y \rightarrow\left(u_{\text {inf }}(t)\right)^{+}} f(t, y), \quad g \text {-a.a. } t \in I \backslash D_{g} .
$$

However, since $u_{\text {inf }}$ is unknown a priori, a reasonable sufficient condition to impose is

$$
\limsup _{y \rightarrow x^{-}} f(t, y) \leq f(t, x) \leq \liminf _{y \rightarrow x^{+}} f(t, y), \quad g \text {-a.a. } t \in I \backslash D_{g}, \forall x \in \mathbb{R} .
$$

\section{Existence of minimal solution}

We start this section with the following lemma, which is an adaptation of [8, Lemma 6.92]. This lemma will be used later to obtain an existence result.

Lemma 4.1 Let $\Phi:[a, b] \rightarrow \mathbb{R}$ be a map such that $\Phi_{g}^{\prime}(t)$ exists for all $t \in E \subset[a, b] \backslash D_{g}$. If $m(\Phi(E))=0$, where $m$ denotes Lebesgue's measure, then $\Phi_{g}^{\prime}(t)=0$ for $g$-a.a. $t \in E$.

Proof Without loss of generality, we can assume that $E \cap\left(C_{g} \cup N_{g}\right)=\emptyset$, where $C_{g}$ can be expressed as the union of pairwise disjoint intervals $C_{g}=\bigcup_{n=1}^{\infty}\left(a_{n}, b_{n}\right)$ and $N_{g}=\left\{a_{n}, b_{n}\right.$ : $n \in \mathbb{N}\} \backslash D_{g}$ (see [4, Remark 2.1]). Let us define the sets

$$
B_{n}=\{t \in E:|\Phi(s)-\Phi(t)|>|g(s)-g(t)| / n, \forall s \in[a, b], 0<s-t<1 / n\}, \quad n \in \mathbb{N},
$$

and $B=\left\{t \in E: \Phi_{g}^{\prime} \neq 0\right\}$. Then $B=\bigcup_{n \in \mathbb{N}} B_{n}$. Indeed, if $t \in B$, then there exists $c>0$ such that

$$
c=\left|\Phi_{g}^{\prime}(t)\right|=\lim _{s \rightarrow t^{+}}\left|\frac{\Phi(s)-\Phi(t)}{g(s)-g(t)}\right| .
$$


Let $\delta>0$ be such that, for $0<s-t<\delta$,

$$
\left|\frac{\Phi(s)-\Phi(t)}{g(s)-g(t)}\right|>\frac{c}{2}
$$

Let $N \in \mathbb{N}$ be such that $1 / N<\min \{\delta, c / 2\}$. Then, for all $n \geq N$, if $0<s-t<1 / n$, it holds that

$$
\left|\frac{\Phi(s)-\Phi(t)}{g(s)-g(t)}\right|<\frac{c}{2}<\frac{1}{n}
$$

and so $t \in B_{n}$ for some $n \in \mathbb{N}$. Conversely, if $t \in B_{n}$ for some $n \in \mathbb{N}$, then $t \in B$ as

$$
\left|\Phi_{g}^{\prime}(t)\right|=\lim _{s \rightarrow t^{+}}\left|\frac{\Phi(s)-\Phi(t)}{g(s)-g(t)}\right|>\frac{1}{n}>0 .
$$

Hence, it is enough to show that $\mu_{g}\left(B_{n}\right)=0$ for all $n \in \mathbb{N}$. Moreover, since each $B_{n}$ can be covered by finitely many intervals of length less than $1 / n$, it suffices to show that $\mu_{g}(J \cap$ $\left.B_{n}\right)=0$ for every such interval $J$. Therefore, if we denote $A=J \cap B_{n}$, we need to show that $\mu_{g}(A)=0$.

Let $\varepsilon>0$. Since $m(\Phi(A))=0$, there exists a family $\left\{J_{k}\right\}_{k=1}^{\infty}$ of open intervals such that

$$
\Phi(A) \subset \bigcup_{k=1}^{\infty} J_{k}, \quad \sum_{k=1}^{\infty}\left|J_{k}\right|<\frac{\varepsilon}{n} .
$$

Let us denote $A_{k}=A \cap \Phi^{-1}\left(J_{k}\right)$. Then $A=\bigcup_{k=1}^{\infty} A_{k}$. Moreover, $g$ - $\operatorname{diam}\left(A_{k}\right) \leq n$. $\operatorname{diam}\left(\Phi\left(A_{k}\right)\right)$. Indeed, by definition $g-\operatorname{diam}\left(A_{k}\right)=\sup \left\{|g(s)-g(t)|: s, t \in A_{k}\right\}$. Therefore, for each pair $s, t \in A_{k}$ such that $s<t$, the definition of $B_{n}$ yields

$$
0<g(s)-g(t)=|g(s)-g(t)|<n|\Phi(s)-\Phi(t)| \leq n \cdot \operatorname{diam}\left(\Phi\left(A_{k}\right)\right)
$$

and so, the inequality follows. Thus, if we prove that $\mu_{g}\left(A_{k}\right) \leq g$ - $\operatorname{diam}\left(A_{k}\right)$, we are done, since

$$
\mu_{g}\left(A_{k}\right) \leq \sum_{k=1}^{\infty} \mu_{g}\left(A_{k}\right) \leq \sum_{k=1}^{\infty} g-\operatorname{diam}\left(A_{k}\right) \leq n \sum_{k=1}^{\infty} \operatorname{diam}\left(\Phi\left(A_{k}\right)\right) \leq n \sum_{k=1}^{\infty}\left|J_{k}\right|<\varepsilon .
$$

To show that $\mu_{g}\left(A_{k}\right) \leq g$ - $\operatorname{diam}\left(A_{k}\right)$, let us denote $a_{k}=\inf A_{k}$ and $b_{k}=\sup A_{k}$. We distinguish two cases: $a_{k} \in A_{k}$ or $a_{k} \notin A_{k}$.

Assume first that $a_{k} \in A_{k}$, then by definition of $b_{k}$, one can find a sequence $\left\{x_{n}\right\}_{n \in \mathbb{N}} \subset A_{k}$ such that $\left\{x_{n}\right\}$ is nondecreasing and $x_{n} \stackrel{n \rightarrow \infty}{\longrightarrow} b_{k}$. Hence,

$$
g-\operatorname{diam}\left(A_{k}\right)=\sup \left\{g(t)-g(s): t, s \in A_{k}, s<t\right\} \geq g\left(x_{n}\right)-g\left(a_{k}\right), \quad \forall n \in \mathbb{N},
$$

and so $g$ - $\operatorname{diam}\left(A_{k}\right) \geq g\left(b_{k}\right)-g\left(a_{k}\right)$. Now, if $b_{k} \in A_{k}$, then $b_{k} \notin D_{g}$ and $A_{k} \subset\left[a_{k}, b_{k}\right]$, so

$$
\mu_{g}\left(A_{k}\right) \leq \mu_{g}\left(\left[a_{k}, b_{k}\right]\right)=g\left(b_{k}^{+}\right)-g\left(a_{k}\right)=g\left(b_{k}\right)-g\left(a_{k}\right) \leq g-\operatorname{diam}\left(A_{k}\right) .
$$

Otherwise $b_{k} \notin A_{k}$, so $A_{k} \subset\left[a_{k}, b_{k}\right)$ and $\mu_{g}\left(A_{k}\right) \leq \mu_{g}\left(\left[a_{k}, b_{k}\right)\right)=g\left(b_{k}\right)-g\left(a_{k}\right) \leq g$ - $\operatorname{diam}\left(A_{k}\right)$. 
Assume now that $a_{k} \notin A_{k}$, then $a_{k}<s<t \leq b_{k}$, and so $g(t)-g(s) \leq g\left(b_{k}\right)-g\left(a_{k}^{+}\right)$. Therefore $g$ - $\operatorname{diam}\left(A_{k}\right) \leq g\left(b_{k}\right)-g\left(a_{k}^{+}\right)$. Moreover, $g$ - $\operatorname{diam}\left(A_{k}\right)=g\left(b_{k}\right)-g\left(a_{k}^{+}\right)$. Indeed, let $\varepsilon^{\prime}>0$. Since $g$ is left-continuous at $b_{k}$, there exists $\delta_{1}>0$ such that if $0 \leq b_{k}-t<\delta_{1}$, then $g\left(b_{k}\right)-g(t)<\varepsilon^{\prime} / 2$. Since $b_{k}=\sup A_{k}$, there exists $t_{0} \in A_{k}$ such that $0 \leq b_{k}-t_{0}<\delta_{1}$, and so $g\left(b_{k}\right)-g\left(t_{0}\right)<\varepsilon^{\prime} / 2$. On the other hand, by definition of $g\left(a_{k}^{+}\right)$, there exists $\delta_{2}>0$ such that if $0<s-a_{k}<\delta_{2}$, then $g(s)-g\left(a_{k}^{+}\right)<\varepsilon^{\prime} / 2$. Since $a_{k}=\inf \left(A_{k}\right)$, there exists $s \in A_{k}$ such that $0<s-a_{k}<\min \left\{\delta_{2}, t_{0}-a_{k}\right\}$. Hence, there exist $s<t, s, t \in A_{k}$ such that

$$
g(t)-g(s)>g\left(b_{k}\right)-\frac{\varepsilon^{\prime}}{2}-g\left(a_{k}^{+}\right)-\frac{\varepsilon^{\prime}}{2}=g\left(b_{k}\right)-g\left(a_{k}^{+}\right)-\varepsilon^{\prime} .
$$

Therefore $g$ - $\operatorname{diam}\left(A_{k}\right)=g\left(b_{k}\right)-g\left(a_{k}^{+}\right)$. Again, if $b_{k} \in A_{k}$, then $b_{k} \notin D_{g}$ and $A_{k} \subset\left(a_{k}, b_{k}\right]$, so

$$
\mu_{g}\left(A_{k}\right) \leq \mu_{g}\left(\left(a_{k}, b_{k}\right]\right)=g\left(b_{k}^{+}\right)-g\left(a_{k}^{+}\right)=g\left(b_{k}\right)-g\left(a_{k}^{+}\right)=g-\operatorname{diam}\left(A_{k}\right) .
$$

Otherwise, $b_{k} \notin A_{k}$, so $A_{k} \subset\left(a_{k}, b_{k}\right)$ and $\mu_{g}\left(A_{k}\right) \leq \mu_{g}\left(\left(a_{k}, b_{k}\right)\right)=g\left(b_{k}\right)-g\left(a_{k}^{+}\right)=$ $g-\operatorname{diam}\left(A_{k}\right)$.

Using Theorems 3.5 and 3.8, we obtain the following existence result.

Theorem 4.2 Let $f:[0,1] \times \mathbb{R} \rightarrow \mathbb{R}$ be a mapping satisfying hypotheses $(\mathrm{H} 1)-(\mathrm{H} 2)$. Moreover, assume that $f$ satisfies the following:

(H3) Either

$$
\limsup _{y \rightarrow x^{-}} f(t, y) \leq f(t, x) \leq \liminf _{y \rightarrow x^{+}} f(t, y), \quad g \text {-a.a. } t \in I \backslash D_{g}, \forall x \in \mathbb{R},
$$

or there exists a family of $g$-absolutely continuous functions $\gamma_{n}:\left[c_{n}, d_{n}\right] \subset I \rightarrow \mathbb{R}$, $n \in \mathbb{N}$, such that, for g-a.a. $t \in I \backslash D_{g}$ and all $x \in \mathbb{R} \backslash \bigcup_{\left\{n \in \mathbb{N}: c_{n} \leq t \leq d_{n}\right\}}\left\{\gamma_{n}(t)\right\}$, inequality (4.1) holds, while for each $n \in \mathbb{N}$ and g-a.a. $t \in\left[c_{n}, d_{n}\right] \backslash D_{g}$, we have either $\left(\gamma_{n}\right)_{g}^{\prime}(t)=f\left(t, \gamma_{n}(t)\right)$ or

$$
\begin{aligned}
& \left(\gamma_{n}\right)_{g}^{\prime}(t) \geq f\left(t, \gamma_{n}(t)\right) \quad \text { whenever }\left(\gamma_{n}\right)_{g}^{\prime}(t) \geq \liminf _{y \rightarrow\left(\gamma_{n}(t)\right)^{+}} f(t, y), \\
& \left(\gamma_{n}\right)_{g}^{\prime}(t) \leq f\left(t, \gamma_{n}(t)\right) \quad \text { whenever }\left(\gamma_{n}\right)_{g}^{\prime}(t) \leq \limsup _{y \rightarrow\left(\gamma_{n}(t)\right)^{-}} f(t, y) .
\end{aligned}
$$

Then:

(a) $\left(u_{\mathrm{inf}}\right)_{g}^{\prime}(t)=f\left(t, u_{\mathrm{inf}}(t)\right)$ for $g$-a.a. $t \in I \backslash J$, where $J$ is a countable union of sets which contain no positive g-measure set. Specifically, $J=\bigcup_{n, m \in \mathbb{N}} J_{n, m}$, where, for all $n, m \in \mathbb{N}$, the set

$$
J_{n, m}=\left\{t \in I \backslash D_{g}:\left(u_{\mathrm{inf}}\right)_{g}^{\prime}(t)-\frac{1}{n}>\sup \left\{f(t, y): u_{\mathrm{inf}}(t)-\frac{1}{m}<y<u_{\mathrm{inf}}(t)\right\}\right\}
$$

contains no positive g-measure set.

(b) $u_{\mathrm{inf}}$ is the minimal solution of (1.1) provided that, for all $n, m \in \mathbb{N}$, the set $J_{n, m}$ is g-measurable. 
Proof We shall assume that the second alternative in (H3) holds, as the proof in the other case is analogous, but simpler. By Theorem 3.5 there exists $I_{1} \subset I$ such that $D_{g} \subset I_{1}$ and

$$
\left(u_{\text {inf }}\right)_{g}^{\prime}(t) \geq f\left(t, u_{\text {inf }}(t)\right) \chi_{I_{1}}(t)+\liminf _{y \rightarrow\left(u_{\text {inf }}(t)\right)^{+}} f(t, y) \chi_{I \backslash I_{1}}(t), \quad g \text {-a.a. } t \in I .
$$

We then deduce from $(\mathrm{H} 3)$ that

$$
\left(u_{\text {inf }}\right)_{g}^{\prime}(t) \geq f\left(t, u_{\text {inf }}(t)\right) \quad \text { holds for } g \text {-a.a. } t \in I \backslash \bigcup_{n \in \mathbb{N}} A_{n} \text {, }
$$

where $A_{n}=\left\{t \in\left[c_{n}, d_{n}\right] \backslash D_{g}: u_{\text {inf }}(t)=\gamma_{n}(t)\right\}$.

For each $n \in \mathbb{N}$, define $\Phi_{n}(t)=u_{\text {inf }}(t)-\gamma_{n}(t), t \in\left[c_{n}, d_{n}\right]$, and

$$
E_{n}=\left\{t \in A_{n}: \exists\left(u_{\mathrm{inf}}\right)_{g}^{\prime}(t),\left(\gamma_{n}\right)_{g}^{\prime}(t)\right\}
$$

Applying Lemma 4.1 with $\Phi=\Phi_{n}$ and $E=E_{n}$, we obtain $\left(u_{\text {inf }}\right)_{g}^{\prime}(t)=\left(\gamma_{n}\right)_{g}^{\prime}(t)$ for $g$-a.a. $t \in E_{n}$. Since $u_{\text {inf }}$ and $\gamma_{n}$ are $g$-absolutely continuous, we have $\mu_{g}\left(A_{n} \backslash E_{n}\right)=0$, hence $\left(u_{\text {inf }}\right)_{g}^{\prime}(t)=$ $\left(\gamma_{n}\right)_{g}^{\prime}(t)$ for $g$-a.a. $t \in A_{n}$. Therefore, (4.5) yields

$$
\left(u_{\text {inf }}\right)_{g}^{\prime}(t) \geq f\left(t, u_{\text {inf }}(t)\right) \quad \text { for } g \text {-a.a. } t \in I \backslash \bigcup_{n \in \mathbb{N}} \Gamma_{n},
$$

where $\Gamma_{n}=\left\{t \in\left[c_{n}, d_{n}\right] \backslash D_{g}: u_{\text {inf }}(t)=\gamma_{n}(t),\left(\gamma_{n}\right)_{g}^{\prime}(t) \neq f\left(t, \gamma_{n}(t)\right)\right\}$.

Let us show that, in fact, the inequality holds for $g$-a.a. $t \in I$. To do so, let $n \in \mathbb{N}$ be fixed, and let $t_{0} \in \Gamma_{n}$ be such that $\left(u_{\text {inf }}\right)_{g}^{\prime}\left(t_{0}\right)=\left(\gamma_{n}\right)_{g}^{\prime}\left(t_{0}\right)$. We study separately two cases: either

$$
\left(\gamma_{n}\right)_{g}^{\prime}\left(t_{0}\right)<\liminf _{y \rightarrow\left(\gamma_{n}\left(t_{0}\right)\right)^{+}} f(t, y) \quad \text { or } \quad\left(\gamma_{n}\right)_{g}^{\prime}\left(t_{0}\right) \geq \liminf _{y \rightarrow\left(\gamma_{n}\left(t_{0}\right)\right)^{+}} f(t, y) .
$$

If $\left(\gamma_{n}\right)_{g}^{\prime}\left(t_{0}\right)<\liminf _{y \rightarrow\left(\gamma_{n}\left(t_{0}\right)\right)^{+}} f(t, y)$, then $\left(u_{\text {inf }}\right)_{g}^{\prime}\left(t_{0}\right)<\liminf _{y \rightarrow\left(\gamma_{n}\left(t_{0}\right)\right)^{+}} f(t, y)$. Hence, by $(4.4)$, either $t_{0}$ belongs to a null-measure set or $t_{0} \in I_{1}$, and so $\left(u_{\text {inf }}\right)_{g}^{\prime}\left(t_{0}\right) \geq f\left(t_{0}, u_{\text {inf }}\left(t_{0}\right)\right)$. Otherwise, $\left(\gamma_{n}\right)_{g}^{\prime}\left(t_{0}\right) \geq \liminf _{y \rightarrow\left(\gamma_{n}\left(t_{0}\right)\right)^{+}} f(t, y)$, and so by (4.2) either $t_{0}$ belongs to a null-measure set or $\left(\gamma_{n}\right)_{g}^{\prime}\left(t_{0}\right) \geq f\left(t_{0}, \gamma_{n}\left(t_{0}\right)\right)$, and therefore $\left(u_{\text {inf }}\right)_{g}^{\prime}\left(t_{0}\right) \geq f\left(t_{0}, u_{\text {inf }}\left(t_{0}\right)\right)$.

We have thus proven that $\left(u_{\text {inf }}\right)_{g}^{\prime}(t) \geq f\left(t, u_{\text {inf }}(t)\right)$ for $g$-a.a. $t \in I$. Now, applying Theorem 3.8, for $g$-a.a. $t \in I \backslash J$, we have either $t \in D_{g}$, and then $\left(u_{\text {inf }}\right)^{\prime}(t)=f\left(t, u_{\text {inf }}(t)\right)$, or $t \notin D_{g}$ and

$$
\left(u_{\mathrm{inf}}\right)_{g}^{\prime}(t) \leq \limsup _{y \rightarrow\left(u_{\mathrm{inf}}(t)\right)^{-}} f(t, y)
$$

Therefore, (4.1) implies that $\left(u_{\text {inf }}\right)_{g}^{\prime}(t) \leq f\left(t, u_{\text {inf }}(t)\right)$ for $g$-a.a. $t \in(I \backslash J) \backslash \bigcup_{n \in \mathbb{N}} A_{n}$. Let us show that the inequality holds for $g$-a.a. $t \in I \backslash J$.

Let $n \in \mathbb{N}$ be fixed. Since $\left(u_{\text {inf }}\right)_{g}^{\prime}=\left(\gamma_{n}\right)_{g}^{\prime} g$-almost everywhere in $A_{n}$, it suffices to see what happens at an arbitrary point $t_{0} \in A_{n}$ such that $\left(u_{\text {inf }}\right)_{g}^{\prime}\left(t_{0}\right)=\left(\gamma_{n}\right)_{g}^{\prime}\left(t_{0}\right)$. Recall that $u_{\text {inf }}\left(t_{0}\right)=$ $\gamma_{n}\left(t_{0}\right)$ and $t_{0} \notin D_{g}$. Now, if $\left(\gamma_{n}\right)_{g}^{\prime}\left(t_{0}\right)>\lim \sup _{y \rightarrow\left(\gamma_{n}\left(t_{0}\right)\right)^{-}} f(t, y)$, then

$$
\left(u_{\text {inf }}\right)_{g}^{\prime}\left(t_{0}\right)>\limsup _{y \rightarrow\left(u_{\text {inf }}\left(t_{0}\right)\right)^{-}} f(t, y)
$$


hence, $t_{0} \in J$. Otherwise, $\left.\left(\gamma_{n}\right)_{g}^{\prime}\left(t_{0}\right) \leq \lim _{\sup } \sin _{n}\left(t_{0}\right)\right)^{-} f(t, y)$, by (4.3), either $t_{0}$ belongs to a null-measure set or $\left(\gamma_{n}\right)_{g}^{\prime}\left(t_{0}\right) \leq f\left(t_{0}, \gamma_{n}\left(t_{0}\right)\right)$, and therefore $\left(u_{\text {inf }}\right)_{g}^{\prime}\left(t_{0}\right) \leq f\left(t_{0}, u_{\text {inf }}\left(t_{0}\right)\right)$.

Hence $\left(u_{\text {inf }}\right)_{g}^{\prime}(t) \leq f\left(t, u_{\text {inf }}(t)\right)$ for $g$-a.a. $t \in I \backslash J$, and so

$$
\left(u_{\text {inf }}\right)_{g}^{\prime}(t)=f\left(t, u_{\text {inf }}(t)\right), \quad g \text {-a.a. } t \in I \backslash J .
$$

Part (b) follows from (a) with the extra assumption.

Part (a) of Theorem 4.2 ensures that $u_{\text {inf }}$ is some sort of "weak" solution, which is an extremely weak concept as a countable union of sets having no positive $g$-measure may be rather big. Anyway, the measurability of the sets $J_{n, m}$ is enough to turn $u_{\text {inf }}$ into a solution.

To conclude this section, we shall prove the result that will give an easily verifiable sufficient condition for the measurability of $J_{n, m}$. In order to do so, we first start by proving two lemmas related to a family of functions $\mathcal{S}_{x}$ that shall be needed later in the proof of the mentioned result.

Lemma 4.3 Let $x: I \rightarrow \mathbb{R}$ be a function of bounded variation. Then there exists a sequence $\left\{x_{n}\right\}_{n=1}^{\infty}$ of step functions such that $x_{n}(t) \in \mathbb{Q}$ for all $t \in I$ and $\left\{x_{n}\right\} \rightarrow x$ uniformly on $I$.

Proof It is enough to show that such a sequence exists for a nondecreasing function $f: I \rightarrow \mathbb{R}$, as any function of bounded variation can be expressed as difference of two nondecreasing functions. Consider the sequence

$$
f_{n}(t):=\frac{1}{n}[n \cdot f(t)]
$$

where $[z]$ denotes the integer part of $z$. First, note that $f_{n}(t) \in \mathbb{Q}$ for all $t \in I$; moreover, each $f_{n}$ is a step function since $f$ is nondecreasing. Therefore, it is enough to show that $\left\|f-f_{n}\right\|_{\infty} \rightarrow 0$ as $n \rightarrow \infty$. Indeed,

$$
\begin{aligned}
0 & \leq\left\|f-f_{n}\right\|_{\infty}=\sup _{t \in I}\left|f_{n}(t)-\frac{1}{n}[n \cdot f(t)]\right|=\frac{1}{n} \sup |n \cdot f(t)-[n \cdot f(t)]| \\
& \leq \frac{1}{n} \stackrel{n \rightarrow \infty}{\longrightarrow} 0 .
\end{aligned}
$$

Given $x \in \mathcal{A C}_{g}(I)$ and $\varepsilon>0$, let us denote by $\left\{x_{n}\right\}$ the sequence obtained from Lemma 4.3 and denote by $D$ the set

$$
D=\bigcup_{n \in \mathbb{N}}\left\{t \in I: x_{n} \text { is not continuous at } t\right\} .
$$

Note that $D$ is a countable set as it is a countable union of countable sets. We define the set $\mathcal{S}_{x}$ as the set of step functions defined as follows: $v:(0,1) \rightarrow \mathbb{R}$ belongs to $\mathcal{S}_{x}$ if, and only if,

1. $x(t)-\varepsilon<v(t)<x(t)$ for all $t \in(0,1)$;

2. $v(t) \in \mathbb{Q}$ for all $t \in(0,1)$;

3. There exist $a_{1}<a_{2}<\cdots<a_{m} \in D$ such that $v$ is constant on $\left(0, a_{1}\right),\left(a_{1}, a_{2}\right), \ldots,\left(a_{m-1}, a_{m}\right),\left(a_{m}, 1\right)$. 
Note that the set $\mathcal{S}_{x}$ is nonempty. Indeed, since $\left\{x_{n}\right\}$ converges uniformly on $I$ to $x$, there exists $N \in \mathbb{N}$ such that

$$
x(t)-\frac{\varepsilon}{3}<x_{N}(t)<x(t)+\frac{\varepsilon}{3}, \quad \forall t \in I .
$$

Define $s(t)=x_{N}(t)-q$ for some $q \in(\varepsilon / 3,2 \varepsilon / 3) \cap \mathbb{Q}$. It is easy to see that $s \in \mathcal{S}$.

Lemma 4.4 Let $x \in \mathcal{A C}_{g}(I)$. For all $t_{0} \in(0,1)$, all $\varepsilon>0$, all $y \in\left(x\left(t_{0}\right)-\varepsilon, x\left(t_{0}\right)\right)$ and all $\delta>0$, there exists $s \in \mathcal{S}_{x}$ such that $y-\delta<s\left(t_{0}\right)<y$. Analogously, for all $t_{0} \in(0,1)$, all $\varepsilon>0$, all $y \in\left(x\left(t_{0}\right)-\varepsilon, x\left(t_{0}\right)\right)$ and all $\delta>0$, there exists $s \in \mathcal{S}_{x}$ such that $y<s\left(t_{0}\right)<y+\delta$.

Proof We shall prove the first part of the statement, as the second part is analogous. Fix $t_{0} \in(0,1), \varepsilon>0, y \in\left(x\left(t_{0}\right)-\varepsilon, x\left(t_{0}\right)\right)$ and $\delta>0$. Take $\tilde{\delta} \in(0, \delta]$ such that $x\left(t_{0}\right)-\varepsilon<y-\tilde{\delta}$. Since $\left\{x_{n}\right\} \rightarrow x$ uniformly on $I$ and $y \in\left(x\left(t_{0}\right)-\varepsilon, x\left(t_{0}\right)\right)$, we can find $j, N \in \mathbb{N}$ big enough so that

$$
x\left(t_{0}\right)-\frac{j-1}{j} \varepsilon<y-\tilde{\delta}<y<x\left(t_{0}\right)-\frac{\varepsilon}{j} \quad \text { and } \quad x(t)-\frac{\varepsilon}{2 j}<x_{N}(t)<x(t)+\frac{\varepsilon}{2 j}, \quad \forall t \in I .
$$

The function $s(t)=x_{N}(t)-x_{N}\left(t_{0}\right)+q$ for some $q \in(y-\tilde{\delta}, y) \cap \mathbb{Q}$ verifies the statement of the lemma. Indeed, first $s \in \mathcal{S}_{x}$ since conditions 2 and 3 are trivially fulfilled and

$$
\begin{aligned}
s(t) & =x_{N}(t)-x_{N}\left(t_{0}\right)+q<x(t)+\frac{\varepsilon}{2 j}-x\left(t_{0}\right)+\frac{\varepsilon}{2 j}+y=x(t)-x\left(t_{0}\right)+\frac{\varepsilon}{j}+y \\
& <x(t)-x\left(t_{0}\right)+\frac{\varepsilon}{j}+x\left(t_{0}\right)-\frac{\varepsilon}{j}=x(t) ; \\
s(t) & =x_{N}(t)-x_{N}\left(t_{0}\right)+q>x(t)-\frac{\varepsilon}{2 j}-x\left(t_{0}\right)-\frac{\varepsilon}{2 j}+y-\tilde{\delta}=x(t)-x\left(t_{0}\right)-\frac{\varepsilon}{j}+y-\tilde{\delta} \\
& >x(t)-x\left(t_{0}\right)-\frac{\varepsilon}{j}+x\left(t_{0}\right)-\frac{j-1}{j} \varepsilon=x(t)-\varepsilon .
\end{aligned}
$$

Moreover, $s\left(t_{0}\right)=x_{N}\left(t_{0}\right)-x_{N}\left(t_{0}\right)+q=q \in(y-\tilde{\delta}, y) \cap \mathbb{Q} \subset(y-\delta, y) \cap \mathbb{Q}$.

The following theorem gives a sufficient condition for $J_{n, m}$ being measurable, and therefore, a useful result to turn $u_{\text {inf }}$ into a solution.

Theorem 4.5 Let $N \subset I$ be a g-null measure set, and let $f: I \times \mathbb{R} \rightarrow \mathbb{R}$ be a function such that $f(\cdot, q)$ is $g$-measurable for each $q \in \mathbb{Q}$. If, for all $t \in I \backslash N$ and all $x \in \mathbb{R}$, we have

$$
\max \left\{\liminf _{y \rightarrow x^{-}} f(t, y), \liminf _{y \rightarrow x^{+}} f(t, y)\right\} \geq f(t, x),
$$

then, for all $x \in \mathcal{A C}_{g}(I)$ and all $\varepsilon>0$, the mapping

$$
t \in I \mapsto \sup \{f(t, y): x(t)-\varepsilon<y<x(t)\}
$$

is g-measurable. 
Proof Fix $x \in \mathcal{A C}_{g}(I)$ and $\varepsilon>0$. Define $\mathcal{S}_{x}$ as before. Then $\mathcal{S}_{x}$ is a countable family of functions. Indeed, since $D$ is countable, the set $D^{m}$ is countable for each $m \in \mathbb{N}$. For each $\omega=\left(\omega_{1}, \ldots, \omega_{m}\right) \in D^{m}$, let us denote by $\mathcal{S}_{\omega}$ a set of step functions of $\mathcal{S}_{x}$ that are constant on the intervals whose extreme points are consecutive numbers of $\omega$. It is easy to see that each $\mathcal{S}_{\omega}$ is countable, and so $\mathcal{S}_{x}$ is countable as it can be written as

$$
\mathcal{S}_{x}=\bigcup_{m \in \mathbb{N}}\left(\bigcup_{\omega \in D^{m}} \mathcal{S}_{\omega}\right)
$$

Hence, given that $f(\cdot, s(\cdot))$ is $g$-measurable on $(0,1)$ for $s \in \mathcal{S}$, it is enough to show that $\sigma=\sigma_{0}$, where

$$
\sigma(t):=\sup _{y \in(x(t)-\varepsilon, x(t))} f(t, y), \quad \sigma_{0}(t):=\sup _{s \in \mathcal{S}} f(t, s(t)) .
$$

It is obvious that $\sigma(t) \geq \sigma_{0}(t)$ on $(0,1)$. To prove that $\sigma_{0} \geq \sigma$ on $(0,1) \backslash N$, fix $t_{0} \in(0,1) \backslash$ $N$ and take a sequence $\left\{y_{n}\right\}_{n \in \mathbb{N}} \subset\left(x\left(t_{0}\right)-\varepsilon, x\left(t_{0}\right)\right)$ such that $\lim _{n \rightarrow \infty} f\left(t_{0}, y_{n}\right)=\sigma\left(t_{0}\right)$. Our assumptions guarantee that, for each $n$, we have that either $\liminf _{y \rightarrow y_{n}^{-}} f\left(t_{0}, y\right) \geq f\left(t_{0}, y_{n}\right)$ or $\liminf _{y \rightarrow y_{n}^{+}} f\left(t_{0}, y\right) \geq f\left(t_{0}, y_{n}\right)$. Assume that the first case holds as the other one is analogous. By definition, we have

$$
f\left(t_{0}, y_{n}\right) \leq \liminf _{y \rightarrow y_{n}^{-}} f\left(t_{0}, y\right)=\lim _{r \rightarrow 0^{+}}\left(\inf _{y_{n}-r<z<y_{n}} f\left(t_{0}, z\right)\right) .
$$

Then there exists $\delta>0$ such that $\inf _{y_{n}-\delta<z<y_{n}} f\left(t_{0}, z\right) \geq f\left(t_{0}, y_{n}\right)-1 / n$. Hence, for each $n \in \mathbb{N}$, by Lemma 4.4, there exists $s_{n} \in \mathcal{S}_{x}$ such that $y_{n}-\delta<s_{n}\left(t_{0}\right)<y_{n}$, and so

$$
f\left(t_{0}, s_{n}\left(t_{0}\right)\right) \geq \inf _{y_{n}-\delta<z<y_{n}} f\left(t_{0}, z\right) \geq f\left(t_{0}, y_{n}\right)-\frac{1}{n} .
$$

Therefore, $\sigma_{0}:=\sup _{s \in \mathcal{S}} f\left(t_{0}, s\left(t_{0}\right)\right) \geq f\left(t_{0}, s_{n}\left(t_{0}\right)\right) \geq f\left(t_{0}, y_{n}\right)-1 / n$. Since this holds for each $n \in \mathbb{N}$,

$$
\sigma_{0}\left(t_{0}\right) \geq \lim _{n \rightarrow \infty}\left(f\left(t_{0}, y_{n}\right)-\frac{1}{n}\right)=\lim _{n \rightarrow \infty} f\left(t_{0}, y_{n}\right)=\sigma\left(t_{0}\right),
$$

and so $\sigma=\sigma_{0}$ on $(0,1) \backslash N$.

\section{Existence of extremal solutions}

One can verify that analogous arguments work for the set of admissible lower solutions:

$$
\mathcal{L}=\left\{l \in \mathcal{A C}_{g}(I): l(0) \leq 0, l_{g}^{\prime}(t) \leq f(t, l(t)) g \text {-a.e. on } I,\left|l_{g}^{\prime}\right| \leq M g \text {-a.e. on } I\right\},
$$

and $l_{\text {sup }}(t)=\sup \{l(t): l \in \mathcal{L}\}$ for all $t \in I$, obtaining analogous results. Hence, combining Theorems 4.2 and 4.5 and their analogues for $l_{\text {sup }}$, one can obtain the following result.

Theorem 5.1 Let $f:[0,1] \times \mathbb{R} \rightarrow \mathbb{R}$ be a mapping satisfying $(\mathrm{H} 1)-(\mathrm{H} 3)$. If $f(\cdot, q)$ is $g$ measurable for all $q \in \mathbb{Q}$ and for $g$-a.a. $t \in I$ and all $x \in \mathbb{R}$, it holds that

$$
\min \left\{\limsup _{y \rightarrow x^{-}} f(t, y), \limsup _{y \rightarrow x^{+}} f(t, y)\right\}
$$




$$
\leq f(t, x) \leq \max \left\{\liminf _{y \rightarrow x^{-}} f(t, y), \liminf _{y \rightarrow x^{+}} f(t, y)\right\},
$$

then $u_{\mathrm{inf}}$ is the maximal solution of (1.1) and $l_{\text {sup }}$ is the minimal one.

Next we illustrate the applicability of Theorem 5.1 in a family of examples with nonmonotone discontinuities accumulating around the initial condition.

Example 5.2 Let $g: \mathbb{R} \rightarrow \mathbb{R}$ be an arbitrary nondecreasing and left-continuous function and $\phi:[0,1] \longrightarrow \mathbb{R}$ be a nondecreasing $g$-absolutely continuous function on $[0,1]$ such that $\phi(0)=0$ (take, for instance, $\phi(t)=\lambda(g(t)-g(0)), \lambda>0$ ).

We shall prove by means of Theorem 5.1 that (1.1) has the minimal and the maximal solutions for

$$
f(t, x)= \begin{cases}2+\sin \left(\left[\frac{1}{x+\phi(t)}\right]\right) & \text { if } t \in I \backslash D_{g} \text { and } x>0, \\ 2 & \text { otherwise, }\end{cases}
$$

where square brackets mean integer part. We remark that $f$ is discontinuous and nonmonotone with respect to $x$ on every neighbourhood of the initial condition.

First, observe that $f(t, x) \in(1,3)$ for all $(t, x) \in I \times \mathbb{R}$, which implies (H1); second, for each fixed $t \in I \cap D_{g}$, we have that $f(t, \cdot)$ is constantly equal to 2 , which implies (H2).

Now for (H3). Since $\phi(t) \geq 0$ for all $t \in I$, we deduce that discontinuities can only occur at points $(t, x)$ such that $x=0$ or

$$
\frac{1}{x+\phi(t)}=n \quad \text { for some } n \in \mathbb{N} \text {. }
$$

Therefore, we define $\gamma_{0}(t)=0$ for all $t \in I$ and, for each $n=1,2, \ldots$,

$$
\gamma_{n}(t)=\frac{1}{n}-\phi(t) \quad \text { for all } t \in[0, I]
$$

Notice that, for each fixed $t \in[0,1]$, the mapping $f(t, \cdot)$ is continuous on $\mathbb{R} \backslash \bigcup_{n=0}^{\infty}\left\{\gamma_{n}(t)\right\}$ (it might also be continuous at some points $x=\gamma_{n}(t)$ for some $n \in \mathbb{N}$, but this is not important). Therefore, for each fixed $t \in[0,1]$, the mapping $f(t, \cdot)$ satisfies (4.1) on $\mathbb{R} \backslash$ $\bigcup_{n=0}^{\infty}\left\{\gamma_{n}(t)\right\}$. It remains to show that the curves $\gamma_{n}, n=0,1, \ldots$, either satisfy the differential equation, or they satisfy (4.2) and (4.3). Given $n=0,1, \ldots, \gamma_{n}$ is nonincreasing, the definition of $g$-derivative yields

$$
\left(\gamma_{n}\right)_{g}^{\prime}(t) \leq 0<1 \leq \min \left\{f\left(t, \gamma_{n}(t)\right), \liminf _{y \rightarrow\left(\gamma_{n}(t)\right)^{+}} f(t, y), \limsup _{y \rightarrow\left(\gamma_{n}(t)\right)^{-}} f(t, y)\right\} \quad \text { for } g \text {-a.a. } t \in[0,1] .
$$

Hence, we have that $\gamma_{n}, n=0,1, \ldots$, satisfies (4.3). Moreover,

$$
\left(\gamma_{n}\right)_{g}^{\prime}(t) \geq \limsup _{y \rightarrow\left(\gamma_{n}(t)\right)^{-}} f(t, y), \quad n=0,1, \ldots
$$

only occurs for $t \in A$ with $\mu_{g}(A)=0$. Therefore, (H3) is satisfied. 
Finally, we check that $f(\cdot, q)$ is $g$-measurable for all $q \in \mathbb{Q}$ and that, for $g$-a.a. $t \in I$ and all $x \in \mathbb{R}$, we have

$$
\min \left\{\limsup _{y \rightarrow x^{-}} f(t, y), \limsup _{y \rightarrow x^{+}} f(t, y)\right\} \leq f(t, x) \leq \max \left\{\liminf _{y \rightarrow x^{-}} f(t, y), \liminf _{y \rightarrow x^{+}} f(t, y)\right\} .
$$

The last part follows from the fact that, for each fixed $t \in[0,1]$, the mapping $f(t, \cdot)$ is continuous from the left at every $x \in \mathbb{R}$. Indeed, this is trivial if $t \in D_{g}$; otherwise, observe that $f(t, x)=2$ for all $x \leq 0, f(t, x)=2$ for $x>\gamma_{1}(t)$ and for $n=1,2, \ldots$, we have

$$
f(t, x)=2+\sin (n) \quad \text { for all } x \in\left(\gamma_{n+1}(t), \gamma_{n}(t)\right], x>0 .
$$

To deduce that $f(\cdot, q)$ is $g$-measurable for each $q \in \mathbb{Q}$, just note that $f(\cdot, q)$ assumes a finite number of different values on corresponding Borel-measurable subsets of $[0,1]$, hence $f(\cdot, q)$ is a Borel-measurable function, which implies that $f(\cdot, q)$ is $g$-measurable since Lebesgue-Stieltjes measures are Borel measures.

\subsection{Applications to difference equations}

Any difference equation of the form

$$
x_{n+1}-x_{n}=f\left(n, x_{n}\right), \quad n=0,1,2, \ldots, N, x_{0} \text { given }
$$

can be expressed as a $g$-differential equation

$$
x_{g}^{\prime}(t)=f(t, x(t)) \quad g \text {-a.a. } t \in I=[0, N], x(0)=x_{0},
$$

where $g(t)=\min \{n \in \mathbb{Z}: n \geq t\}$. Indeed, given a solution of (5.2) $x \in \mathcal{A C}_{g}(I)$, and bearing in mind that $C_{g}=I \backslash \mathbb{Z}$ and $D_{g}=\mathbb{Z}$, for all $n \geq 1$, we have

$$
x_{g}^{\prime}(n)=\frac{x\left(n^{+}\right)-x(n)}{g\left(n^{+}\right)-g(n)}=x(n+1)-x(n),
$$

and so $x_{n+1}-x_{n}=x_{g}^{\prime}(n)=f(n, x(n))=f\left(n, x_{n}\right)$. Conversely, if $x: I \cap \mathbb{Z} \rightarrow \mathbb{R}$ is a solution of (5.1), we define $\tilde{x}(t)=x(g(t))$ for all $t \in I$. First of all, note that $\tilde{x} \in \mathcal{A C}_{g}(I)$ since $\tilde{x}_{g}^{\prime}$ exists $g$-a.e. $I$ since for $n \in D_{g}=I \backslash C_{g}$,

$$
\tilde{x}_{g}^{\prime}(n)=\frac{x\left(g\left(n^{+}\right)\right)-x(g(n))}{g\left(n^{+}\right)-g(n)}=x(n+1)-x(n) \in \mathbb{R}
$$

and, moreover, $\tilde{x}_{g}^{\prime} \in \mathcal{L}_{g}^{1}(I)$ as

$$
\int_{I}\left|\tilde{x}_{g}^{\prime}\right| d g=\sum_{i=0}^{N}\left|\tilde{x}_{g}^{\prime}(i)\right| \Delta g(i)=\sum_{i=0}^{N}|x(i+1)-x(i)|<\infty .
$$

Finally, for $t \in I$ fixed, $t \in\left[t_{k}, t_{k+1}\right)$ for some $k=0,1,2, \ldots, N$,

$$
\tilde{x}(0)+\int_{[0, t)} \tilde{x}_{g}^{\prime} d g=x(0)+\sum_{\{i \in I \cap \mathbb{Z}: i<t\}} \tilde{x}_{g}^{\prime}(i) \Delta g(i)=x(0)+\sum_{\{i \in I \cap \mathbb{Z}: i<t\}}(x(i+1)-x(i))
$$




$$
=x(k)=\tilde{x}(t) .
$$

Then it follows from (5.3) that $\tilde{x}$ is a solution of (5.2).

Recalling Remark 3.6, $f$ satisfying conditions $(\mathrm{H} 1)-(\mathrm{H} 2)$ was enough to guarantee that $\left(u_{\text {inf }}\right)_{g}^{\prime}(t)=f\left(t, u_{\text {inf }}(t)\right)$ for $t \in I \cap D_{g}$. Then, if there exists $M: I \cap \mathbb{Z} \rightarrow \mathbb{R}$ such that $|f(n, x)| \leq$ $M(n)$ for all $n \in I \cap \mathbb{Z}$, all $x \in \mathbb{R}$ and for all $n \in I \cap \mathbb{Z}$ the mapping $u \in \mathbb{R} \mapsto u+f(n, u)$ is nondecreasing, we can assure that $u_{\text {inf }}$ is the maximal solution of (5.1). Note that this problem has a unique solution trivially; however, we have proved that such a solution is the infimum of all the upper solutions of the problem. Analogous arguments work for $l_{\text {sup }}$.

\subsection{Applications to impulsive differential equations}

It has been shown in [7] that an impulsive problem of the form

$$
\begin{cases}x^{\prime}(t)=f(t, x(t)) & \text { for a.a. } t \in I \backslash J, \\ x\left(t^{+}\right)=x(t)+I_{t}(x(t)) & \text { if } t \in J,\end{cases}
$$

where $J=\left\{t_{k} \in I: k \in \mathbb{N}\right\}$, can be treated as a Stieltjes differential equation of the form $x_{g}^{\prime}(t)=F(t, x(t))$, where

$$
g(t)=t+\sum_{\left\{k \in \mathbb{N}: t_{k}<t\right\}} 2^{-k}, \quad F(t, x)= \begin{cases}f(t, x) & \text { if } t \in I \backslash J, \\ 2^{k} I_{t_{k}}(x) & \text { if } t \in J, t=t_{k} .\end{cases}
$$

Then, using Theorem 5.1, one can obtain a result assuring the existence of extremal solutions for impulsive differential equations.

Corollary 5.3 Consider (5.4). Suppose that the following conditions are satisfied:

1. $f$ is $\mathcal{L}^{1}(I)$-bounded and, for each $k \in \mathbb{N}$, there exists $\alpha_{k} \in \mathbb{R}$ such that $\left|I_{t_{k}}\right| \leq \alpha_{k}$;

2. For all $k \in \mathbb{N}$, the map $u \in \mathbb{R} \mapsto u+I_{t_{k}}(u)$ is nondecreasing;

3. Either

$$
\limsup _{y \rightarrow x^{-}} f(t, y) \leq f(t, x) \leq \liminf _{y \rightarrow x^{+}} f(t, y), \quad \text { a.a. } t \in I, \forall x \in \mathbb{R},
$$

or there exists a family of functions $\gamma_{n}:\left[a_{n}, b_{n}\right] \subset I \rightarrow \mathbb{R}, n \in \mathbb{N}$, with the following properties:

(i) $\gamma_{n}^{\prime}$ exists for a.a. $t \in I$ and $\gamma_{n}^{\prime} \in \mathcal{L}^{1}(I)$;

(ii) for all $k \in \mathbb{N}, \gamma_{n}\left(t_{k}^{+}\right)$exists and $\sum_{k \in \mathbb{N}}\left|\gamma_{n}\left(t_{k}^{+}\right)-\gamma_{n}\left(t_{k}\right)\right|<\infty$;

(iii) for all $t \in I$,

$$
\gamma_{n}(t)=\gamma_{n}(0)+\int_{[0, t)} \gamma_{n}^{\prime}(s) d s+\sum_{t_{k} \in[0, t)}\left(\gamma_{n}\left(t_{k}^{+}\right)-\gamma_{n}\left(t_{k}\right) ;\right.
$$

(iv) for a.a. $t \in I$ and all $x \in \mathbb{R} \backslash \bigcup_{\left\{n \in \mathbb{N}: a_{n} \leq t \leq b_{n}\right\}}\left\{\gamma_{n}(t)\right\}$, inequality (5.5) holds, while for each $n \in \mathbb{N}$ and a.a. $t \in\left[a_{n}, b_{n}\right]$, we have either $\left(\gamma_{n}\right)^{\prime}(t)=f\left(t, \gamma_{n}(t)\right)$ or

$$
\left(\gamma_{n}\right)^{\prime}(t) \geq f\left(t, \gamma_{n}(t)\right) \quad \text { whenever }\left(\gamma_{n}\right)^{\prime}(t) \geq \liminf _{y \rightarrow\left(\gamma_{n}(t)\right)^{+}} f(t, y)
$$




$$
\left(\gamma_{n}\right)^{\prime}(t) \leq f\left(t, \gamma_{n}(t)\right) \quad \text { whenever }\left(\gamma_{n}\right)^{\prime}(t) \leq \limsup _{y \rightarrow\left(\gamma_{n}(t)\right)^{-}} f(t, y)
$$

4. For all $q \in \mathbb{Q}$, the map $f(\cdot, q)$ is Borel-measurable;

5. For almost all $t \in I \backslash J$ and all $x \in \mathbb{R}$,

$$
\begin{aligned}
& \quad \min \left\{\limsup _{y \rightarrow x^{-}} f(t, y), \limsup _{y \rightarrow x^{+}} f(t, y)\right\} \leq f(t, x) \leq \max \left\{\liminf _{y \rightarrow x^{-}} f(t, y), \liminf _{y \rightarrow x^{+}} f(t, y)\right\} \\
& \text { and for all } k \in \mathbb{N} \text { and all } x \in \mathbb{R}, \\
& \qquad \min \left\{\limsup _{y \rightarrow x^{-}} I_{t_{k}}(y), \underset{y \rightarrow x^{+}}{\limsup } I_{t_{k}}(y)\right\} \leq I_{t_{k}}(x) \leq \max \left\{\liminf _{y \rightarrow x^{-}} I_{t_{k}}(y), \liminf _{y \rightarrow x^{+}} I_{t_{k}}(y)\right\} .
\end{aligned}
$$

Then $u_{\mathrm{inf}}$ is the maximal solution of (5.4) and $l_{\mathrm{sup}}$ is the minimal one.

\section{Acknowledgements}

The authors would like to thank the anonymous referees for their encouraging reports and comments which helped to improve this manuscript.

\section{Funding}

Rodrigo López Pouso was partially supported by Ministerio de Economía y Competitividad, Spain, and FEDER, Project MTM2016-75140-P and Xunta de Galicia under grant ED431C 2019/02. Ignacio Márquez Albés was supported by Ministerio de Economía y Competitividad, Spain, and FEDER, Project MTM2016-75140-P and Xunta de Galicia under grants ED481A-2017/095 and ED431C 2019/02.

\section{Availability of data and materials}

Not applicable.

\section{Competing interests}

The authors declare that they have no competing interests.

\section{Authors' contributions}

All authors contributed equally to the different parts of the manuscript. All authors read and approved the final manuscript.

\section{Publisher's Note}

Springer Nature remains neutral with regard to jurisdictional claims in published maps and institutional affiliations.

Received: 9 October 2019 Accepted: 13 February 2020 Published online: 24 February 2020

\section{References}

1. Antunes Monteiro, G., Slavík, A.: Extremal solutions of measure differential equations. J. Math. Anal. Appl. 444, 568-597 (2016). https://doi.org/10.1016/j.jmaa.2016.06.035

2. Frigon, M., López Pouso, R.: Theory and applications of first-order systems of Stieltjes differential equations. Adv. Nonlinear Anal. 6(1), 13-36 (2017)

3. Graves, L.M.: The Theory of Functions of Real Variables. McGraw-Hill, New York (1946)

4. López Pouso, R.: Necessary conditions for solving initial value problems with infima of superfunctions. Math. Inequal. Appl. 8(4), 633-641 (2005)

5. López Pouso, R., Márquez Albés, I.: General existence principles for Stieltjes differential equations with applications to mathematical biology. J. Differ. Equ. 264, 5388-5407 (2018)

6. López Pouso, R., Márquez Albés, I., Monteiro, G.A.: Extremal solutions of systems of measure differential equations and applications in the study of Stieltjes differential problems. Electron. J. Qual. Theory Differ. Equ. 2018, 38 (2018)

7. López Pouso, R., Rodríguez, A.: A new unification of continuous, discrete, and impulsive calculus through Stieltjes derivatives. Real Anal. Exch. 40(2), 1-35 (2014)

8. Stromberg, K.R.: Introduction to Classical Real Analysis. Wadsworth, Belmont (1981) 